David T. Gay · Margaret Symington

\title{
Toric structures on near-symplectic 4-manifolds
}

Received September 27, 2006

\begin{abstract}
A near-symplectic structure on a 4-manifold is a closed 2-form that is symplectic away from the 1-dimensional submanifold along which it vanishes and that satisfies a certain transversality condition along this vanishing locus. We investigate near-symplectic 4-manifolds equipped with singular Lagrangian torus fibrations which are locally induced by effective Hamiltonian torus actions. We show how such a structure is completely characterized by a singular integral affine structure on the base of the fibration whenever the vanishing locus is nonempty. The base equipped with this geometric structure generalizes the moment map image of a toric 4-manifold in the spirit of earlier work by the second author on almost toric symplectic 4-manifolds. We use the geometric structure on the base to investigate the problem of making given smooth torus actions on 4-manifolds symplectic or Hamiltonian with respect to near-symplectic structures and to give interesting constructions of structures which are locally given by torus actions but have nontrivial global monodromy.
\end{abstract}

Keywords. Symplectic, near-symplectic, toric, torus action, 4-manifold, Hamiltonian, Lagrangian fibration

\section{Introduction}

Advances in symplectic topology in the last decade have shown that symplectic 4-manifolds populate a vast portion of the world of smooth 4-manifolds, extending far beyond the class of Kähler manifolds (see [5, 7] among others). Meanwhile, some of the most powerful techniques for studying them are motivated by complex algebraic geometry. For a 2-form $\omega$ on a $2 n$-dimensional manifold to be symplectic, it must be nondegenerate (i.e. $\omega^{n}$ must be nonvanishing) and it must be closed (i.e. $d \omega=0$ ). The first condition guarantees that the manifold admits an almost complex structure, while the additional condition of closedness allows one to get control of solutions to differential equations involving an "approximate Cauchy-Riemann" operator and obtain compact moduli spaces of solutions.

D. T. Gay: Department of Mathematics and Applied Mathematics, University of Cape Town, Private Bag X3, Rondebosch 7701, South Africa; e-mail: dgay@maths.uct.ac.za

M. Symington: Department of Mathematics, Mercer University, 1400 Coleman Ave., Macon, GA 31207, USA; e-mail: symington_mf@mercer.edu

Mathematics Subject Classification (2000): Primary 57R17; Secondary 53D20, 57M60, 57M50 
Currently it is becoming apparent that one can study a more general class of oriented smooth 4-manifolds using the techniques of pseudo-holomorphic curves [19] and Lefschetz fibrations [3]. Indeed, it suffices that there be a cohomology class whose square induces the given orientation of the 4-manifold $X$, i.e. $b_{2}^{+}(X)>0$. Honda [8] showed that on such a manifold there always exists what is now known as a near-symplectic form:

Definition 1.1. On a smooth, oriented 4-manifold $X$, consider a closed 2-form $\omega$ such that $\omega^{2} \geq 0$ and let $Z_{\omega}$ denote the vanishing locus, the set of points where $\omega=0$. The form $\omega$ is near-symplectic if

(1) $\omega^{2}>0$ on the complement of $Z_{\omega}$

(2) at each point $x \in Z_{\omega}$, if we use local coordinates on a neighborhood $U$ of $x$ to identify the map $\omega: U \rightarrow \Lambda^{2}\left(T^{*} U\right)$ as a smooth map $\omega: \mathbb{R}^{4} \rightarrow \mathbb{R}^{6}$, then its linearization at $x, D \omega_{x}: \mathbb{R}^{4} \rightarrow \mathbb{R}^{6}$, has rank 3 .

We call the set $X \backslash Z_{\omega}$ the symplectic locus. A near-symplectic manifold is an oriented smooth 4-manifold equipped with a near-symplectic form.

Definition 1.1 is a rephrasing, in local coordinates, of the definition of a near-symplectic form given in [3]. Indeed, if $\phi: \mathbb{R}^{4} \rightarrow \mathbb{R}^{4}$ is a change of coordinates on $U$ and $\Phi$ : $\mathbb{R}^{6} \rightarrow \mathbb{R}^{6}$ is the corresponding change of coordinates on $\Lambda^{2}\left(T^{*} U\right)$, then, restricted to $Z_{\omega}, D \omega_{x} \circ \phi=\Phi \circ D \omega_{x}$. Consequently, on $Z_{\omega}$ the map $D \omega_{x}$ represents an intrinsically defined derivative, denoted in [3] by $\nabla \omega_{x}: T_{x} X \rightarrow \Lambda^{2}\left(T_{x}^{*} X\right)$. The same paper explains why 3 is the maximum possible rank for $D \omega_{x}$ and that this definition is equivalent to the original definition (see [8]) in terms of metric properties. Specifically, a closed 2-form $\omega$ on a 4-manifold $X$ is near-symplectic if it is self-dual with respect to some metric $g$ and, viewed as a section of the bundle $\Lambda_{2}^{+}$of $g$-self-dual 2-forms, is transverse to the zero section.

Several threads of emerging research indicate that one should be able to understand the moduli spaces of pseudo-holomorphic curves in near-symplectic manifolds and that the extra structure of a fibration induced by a Hamiltonian torus action should aid in this endeavor. Specifically, Taubes has made initial steps in his program to develop GromovWitten invariants for near-symplectic manifolds that should be invariants of the underlying smooth structure [19, 20]; Mikhalkin has calculated, via tropical algebraic geometry, the Gromov-Witten invariants of toric surfaces in terms of 1-complexes in their moment map images [14]; and Parker has used symplectic field theory to gain an understanding of moduli spaces of pseudo-holomorphic curves in $T^{*} T^{2}$ in terms of 1-complexes in $\mathbb{R}^{2}$ [17]. Presuming success on these fronts, the results in this paper lead one to expect to be able to calculate Taubes' invariants for locally toric near-symplectic manifolds by counting 1-complexes suitably immersed in the bases of the induced fibrations.

This paper can usefully be read in parallel with the work of Kaufman [10], which also develops a theory of toric structures on near-symplectic manifolds.

We begin by giving a characterization of Hamiltonian torus actions on symplectic 4-manifolds that is convenient for generalizing to the near-symplectic setting and that emphasizes the induced fibration. 
The following proposition can be read as a definition by those unfamiliar with Hamiltonian group actions, but is really a statement that a certain characterization of "Hamiltonian" is equivalent to the standard definition (see Definition A.1).

Proposition 1.2. A smooth torus action $\sigma: T^{2} \times X \rightarrow X$ on a symplectic 4-manifold $(X, \omega)$ is Hamiltonian if and only if there exists a smooth map $\mu: X \rightarrow \mathbb{R}^{2}$, called the moment map, such that, for any $\xi \in \mathbb{R}^{2}$, the vector field $V_{\xi}$ whose flow is $x \mapsto \sigma(t \xi, x)$ $($ for $t \in \mathbb{R})$ is defined by the equation $\omega\left(V_{\xi}, W\right)=-\xi \cdot D \mu(W)$ for all $W \in T X$.

A proof of this proposition is provided for convenience in the appendix. Note that this proposition is true only for torus actions. Indeed, the proof relies on the fact that the group is both abelian and compact.

Remark 1.3. As part of the proof of Proposition 1.2 we establish that $\omega\left(V_{\xi}, V_{\eta}\right)=0$ for all $\xi, \eta \in \mathbb{R}^{2}$. This implies that preimages of the moment map are isotropic, and in particular the top-dimensional preimages are Lagrangian.

Generalizing to the near-symplectic setting we have:

Definition 1.4. A smooth torus action $\sigma: T^{2} \times X \rightarrow X$ on a near-symplectic 4-manifold $(X, \omega)$ which preserves $\omega$ is Hamiltonian if there exists a smooth map $\mu: X \rightarrow \mathbb{R}^{2}$ such that $\left.\mu\right|_{X \backslash Z_{\omega}}$ is a moment map for $\left.\sigma\right|_{X \backslash Z_{\omega}}$. In particular, $\left.\sigma\right|_{X \backslash Z_{\omega}}$ is Hamiltonian in the usual sense. We call $\mu: X \rightarrow \mathbb{R}^{2}$ the moment map for $\sigma$. An action is locally Hamiltonian if every orbit has an open neighborhood in which the action is Hamiltonian.

The assumption of the smoothness of $\mu$ is not constraining. Indeed, given any moment map $\mu: X \backslash Z_{\omega} \rightarrow \mathbb{R}^{2}$ for the restriction of a smooth torus action to the symplectic locus of a near-symplectic manifold $(X, \omega)$, it can be shown that $\mu$ extends smoothly across $Z_{\omega}$.

Two questions we address in this paper are:

Question 1.5. Which closed $T^{2}$-manifolds (4-manifolds equipped with smooth effective torus actions) admit near-symplectic forms with respect to which the actions are Hamiltonian?

Question 1.6. Which closed $T^{2}$-manifolds admit near-symplectic forms with respect to which the actions are locally Hamiltonian?

Recall that a toric manifold is a symplectic manifold equipped with an effective Hamiltonian torus action (i.e. only the identity acts trivially). This definition generalizes immediately to near-symplectic manifolds.

We are particularly interested in the fibrations induced by Hamiltonian and locally Hamiltonian torus actions. These structures, rather than the actions themselves, appear to be useful for the study of pseudo-holomorphic curves [17]. Furthermore, shifting the focus to fibrations, there is no need for the manifold to admit a global effective torus action, thereby extending the scope of our results. Therefore we make the following definitions.

Definition 1.7. The boundary of a smooth surface has corners if its boundary is piecewise smooth and each nonsmooth point of the boundary has a neighborhood that smoothly surjects onto a neighborhood of the vertex of a sector in $\mathbb{R}^{2}$. 
Definition 1.8. Let $(X, \omega)$ be a near-symplectic manifold. A toric fibration of a nearsymplectic manifold is a smooth surjective map $\pi: X \rightarrow B$ to a smooth surface with boundary and corners such that the top-dimensional fibers of $\pi$ are Lagrangian tori and, ignoring the smooth structure on $B$, the map $\pi$ is the orbit space projection for an effective Hamiltonian torus action on $(X, \omega)$. A locally toric fibration of $(X, \omega)$ is a smooth surjective map $\pi: X \rightarrow B$ to a smooth surface with boundary and corners such that each fiber has an open neighborhood in which the fibration is toric for some Hamiltonian torus action on the neighborhood. A (locally) toric near-symplectic manifold is a near-symplectic manifold equipped with a (locally) toric fibration.

These generalizations lead us to the following question:

Question 1.9. Which smooth 4-manifolds with $b_{2}^{+}>0$ can be equipped with nearsymplectic forms so as to admit locally toric fibrations?

Our central results (Theorems 3.5 and 3.7) assert that toric near-symplectic structures are in one-to-one correspondence with surfaces equipped with certain singular integral affine structures. (See Definition 3.3). This result generalizes naturally to locally toric near-symplectic manifolds (Theorems 5.2 and 5.4. These theorems allow us to answer questions about realizing smooth torus actions in the near-symplectic world and give a number of interesting constructions of near-symplectic 4-manifolds.

For simply connected manifolds we can answer Questions 1.5, 1.6 and 1.9 concisely:

Theorem 1.10. Every locally toric fibration of a simply connected near-symplectic manifold is toric.

Every smooth effective torus action on a simply connected 4-manifold $X$ with $b_{2}^{+}(X)$ $>0$ is Hamiltonian with respect to some near-symplectic structure. Furthermore, the vanishing locus for any such near-symplectic structure must have exactly $b_{2}^{+}(X)-1$ components.

In [16], which we use extensively here, it is shown that the simply connected $T^{2}$-manifolds are precisely $S^{4}, S^{2} \times S^{2}$, and all connected sums of $\mathbb{C} P^{2}$ and $\overline{\mathbb{C} P^{2}}$. Of course, to support a near-symplectic structure the manifold must either be $S^{2} \times S^{2}$ or have at least one $\mathbb{C} P^{2}$ summand to make $b_{2}^{+}>0$.

To state other results requires the notion of positive turning along boundary components of the orbit space of a torus action (Definition 4.5). Briefly, each boundary component of the orbit space is naturally decomposed as a union of edges, to each of which is associated a "slope" in $\mathbb{Q} \cup\{\infty\}$, corresponding to the stabilizer subgroups of orbits above the segments (as shown in [16]). The orbit space, together with this decomposition, is the weighted orbit space. As one traverses a boundary component, the positive turning is the total angular turning of these slopes, where from one edge to the next one turns always counterclockwise. Because each boundary component is a closed curve, this number is always a nonnegative integral multiple of $\pi$.

The following proposition shows how the notion of positive turning can be used to recognize the topological type of a $T^{2}$-manifold from its orbit space. Accordingly, this result is complementary to those in [16]. 
Proposition 1.11. Consider a simply connected $T^{2}$-manifold $(X, \sigma)$ whose weighted orbit space has more than four edges. Let $T$ be the positive turning of the weighted orbit space and let $V$ be the number of its vertices. Then $X$ is diffeomorphic to $m \mathbb{C} P^{2} \# n \overline{\mathbb{C} P}^{2}$ where $m=T / \pi-1$ and $n=V-m-2$.

Note that when there are four or fewer edges, the topology can be determined merely by comparing the weighted orbit space with a short set of examples.

Theorem 1.12. Consider a closed $T^{2}$-manifold $(X, \sigma)$ such that $\sigma$ has no nontrivial $f$ nite stabilizers. Let $T_{0}$ be the largest positive turning along any of the boundary components of the orbit space $B$. Then there exist near-symplectic forms with respect to which $\sigma$ is Hamiltonian only if $T_{0} \geq 2 \pi$. If $g=0$ then having $T_{0} \geq 2 \pi$ is in fact sufficient to give the existence of such a near-symplectic form.

Meanwhile, given any compact surface with nonempty boundary, there is a toric nearsymplectic manifold with orbit space $B$.

Question 1.13. In the preceding theorem, when $g>0$, is there a clean statement of a necessary and sufficient condition for making the action Hamiltonian?

The second author is investigating this question. In the meantime, we can provide necessary and sufficient conditions for making the action locally Hamiltonian:

Theorem 1.14. Consider a closed $T^{2}$-manifold $(X, \sigma)$ whose action $\sigma$ has no nontrivial finite stabilizers. Let $g$ and $k$ be the genus and Euler characteristic of the orbit space $B$. Let $T$ be the sum of the positive turnings along all of the boundary components of the weighted orbit space. Then there exist near-symplectic forms on $X$ with respect to which $\sigma$ is locally Hamiltonian if and only if either

(1) B is a torus, or

(2) B has nonempty boundary and either

(a) $g \geq 1$,

(b) $g=0$ and $T \geq(3-k) \pi$, or

(c) $B$ is an annulus, $T=0$, and the two boundary components each have one edge of the same slope.

The number of components of the vanishing locus must be $(T-2 \pi \chi) / \pi$ where $\chi=$ $2-2 g-k$ is the Euler characteristic of the surface.

Theorem 1.14 implies that, up to homeomorphism, any surface with nonempty boundary is the orbit space of a near-symplectic manifold with a torus action that is locally Hamiltonian. Furthermore, there are no constraints on the one-dimensional stabilizer subgroups unless $B$ is a disk or an annulus.

A locally toric fibration can arise from a global torus action only if the integral affine monodromy of the base (Definition 5.3) is trivial. With regard to locally toric fibrations not induced by global torus actions we show: 
Proposition 1.15. Given any compact surface with boundary $B$ and any homomorphism $h: \pi_{1}(B) \rightarrow G L(2, \mathbb{Z})$, there is a closed near-symplectic manifold $(X, \omega)$ that admits a locally toric fibration with base $B$ and monodromy $h$. (Here, as part of the construction, $B$ inherits a smooth structure.)

Proposition 1.16. There is an infinite family of mutually nondiffeomorphic closed nearsymplectic manifolds that support locally toric structures with nontrivial monodromy, none of which are diffeomorphic to a locally toric near-symplectic manifold with trivial monodromy.

The general philosophy in this paper, thanks to Theorems 3.5, 3.7, 5.2 and 5.4, is to study toric and locally toric near-symplectic manifolds in terms of immersed polygons in the plane that represent the base, or a fundamental domain of the base, of the induced fibration.

The bases of toric and locally toric fibrations carry a naturally defined geometry whose local isometries belong to $\operatorname{Aff}(2, \mathbb{Z}):=\left\{p \mapsto A p+b \mid A \in G L(2, \mathbb{Z}), b \in \mathbb{R}^{2}\right\}$, namely an integral affine structure. Isometric immersions of such surfaces into the plane, equipped with the standard integral affine structure (Definition 3.1), are such that almost every point has a neighborhood whose image is equivalent, up to the action of an element of $\operatorname{Aff}(2, \mathbb{Z})$, to a domain in the first quadrant $Q$ of $\mathbb{R}^{2}$. At the remaining points, all of which are on the boundary, singularities are allowed where the boundary can double back on itself as in Figure 1
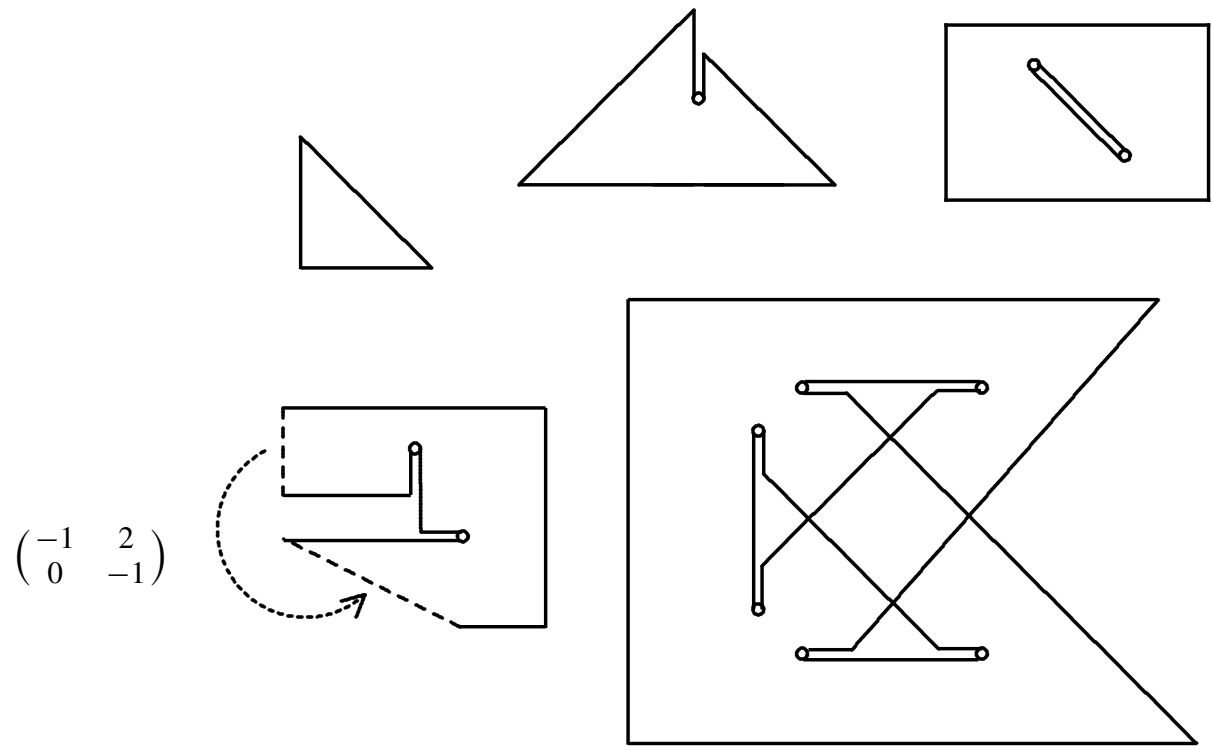

Fig. 1. Various examples of integral affine surfaces, with and without edge folds. 
Example 1.17. To conclude this introduction, Figure 1 gives an indication of the variety of integral affine surfaces with edge folds, and hence of locally toric near-symplectic manifolds. Each open circle represents a component of the vanishing locus. The parts of an edge that limit onto an open circle are drawn slightly displaced as a visual aid, although they should be understood to coincide. By Theorem 5.4 each of these surfaces determines a unique locally toric near-symplectic manifold.

All but one of the figures is the image in $\left(\mathbb{R}^{2}, \mathcal{A}_{0}\right)$ of the base of a locally toric fibration via an immersion that preserves integral affine structures. The figure with the dotted lines is the image under such an immersion of the complement of a line segment, across which there is nontrivial monodromy. To reconstruct the surface, identify the dotted edges by the element of $\operatorname{Aff}(2, \mathbb{Z})$ determined by the linear map indicated and an appropriate translation.

From left to right in the top row, the first figure is the usual moment map image of $\mathbb{C} P^{2}$ while the second is an integral affine base of $\mathbb{C} P^{2} \# \mathbb{C} P^{2}$. (Seeing this would be a good exercise to test one's understanding of Section 3 .) The bottom right figure is the immersed image of a surface of genus one with one boundary component. It can be modified easily to give examples with higher genus or more boundary components.

\section{Local models}

We devote this section to models for the neighborhoods of orbits in a near-symplectic manifold equipped with an effective Hamiltonian torus action. There are four types of orbits that can appear in this setting, characterized by the dimension of the stabilizer subgroup and whether or not the orbit belongs to the vanishing locus.

In general, for a smooth torus action on a 4-manifold, the possible stabilizer subgroups are the identity, a circle subgroup, the whole torus, or a nontrivial finite subgroup [16]. Following [16] we denote circle subgroups (stabilizers of circle orbits) by $G_{(a, b)}:=\left\{\left(t_{1}, t_{2}\right) \mid\left(t_{1}, t_{2}\right) \cdot(a, b)=0\right\}$. Here and throughout this paper, $\left(t_{1}, t_{2}\right)$ are $\mathbb{R} / 2 \pi \mathbb{Z}$-valued coordinates on the 2-dimensional Lie group $T^{2}=S^{1} \times S^{1}$.

An important feature of a Hamiltonian torus action is that the orbits are isotropic (i.e., the symplectic form evaluates trivially on pairs of vectors tangent to an orbit). Hence, for an effective Hamiltonian torus action on a 4-manifold, the generic orbits are Lagrangian tori (isotropic and half the dimension of the ambient manifold). Arnold's Theorem [1] asserts that Lagrangian torus orbits have a standard product neighborhood, and thereby prevents the presence of orbits with nontrivial finite stabilizer. Therefore, in the symplectic locus there are isolated point fibers, circle fibers that come in one-dimensional families, and the generic torus orbits. Meanwhile, because a Hamiltonian action on a near-symplectic manifold preserves the near-symplectic form, the vanishing locus must be a union of orbits. The fact that point orbits are isolated then implies that each component of the vanishing locus is one circle orbit.

We will see that the following two examples provide all the local information about Hamiltonian torus actions on near-symplectic manifolds. 
Example 2.1 (Standard torus action on $\mathbb{R}^{4}$ ). Convenient coordinates on $\mathbb{R}^{4}=\mathbb{R}^{2} \times \mathbb{R}^{2}$ are the square polar coordinates $(p, q):=\left(p_{1}, q_{1}, p_{2}, q_{2}\right)$ which, with respect to polar coordinates $(r, \theta)$, are given by $p=\frac{1}{2} r^{2}, q=\theta$. Then the standard symplectic structure on $\mathbb{R}^{4}$, which with respect to Euclidean coordinates $(x, y)$ is $\omega_{0}=d x \wedge d y:=d x_{1} \wedge$ $d y_{1}+d x_{2} \wedge d y_{2}$, takes the form $\omega_{0}=d p \wedge d q:=d p_{1} \wedge d q_{1}+d p_{2} \wedge d q_{2}$. On $\left(\mathbb{R}^{4}, \omega_{0}\right)$ we have the standard torus action given by

$$
t \cdot(p, q)=(p, q+t)
$$

and the corresponding standard moment map $\mu_{0}: \mathbb{R}^{4} \rightarrow Q=\{(x, y) \mid x \geq 0, y \geq 0\}$ given by $\mu_{0}(p, q)=p$, so $x=p_{1}$ and $y=p_{2}$.

The closed first quadrant $Q$ is the orbit space of this action. For the reader unfamiliar with toric manifolds, we point out that:

(1) The vertex of $Q$ is the image of a point orbit, with torus stabilizer.

(2) A nonvertex point on the boundary of $Q$ is the image of a circle orbit with stabilizer $G_{(1,0)}$ or $G_{(0,1)}$ depending on whether the point is in the positive $x$-axis or positive $y$-axis, respectively.

(3) A point in the interior of $Q$ is the image of a torus orbit, with trivial stabilizer.

Example 2.2 (Standard toric action near the vanishing locus). Following [6] we construct a model neighborhood of a component of the vanishing locus in a near-symplectic manifold as follows: Let $\alpha \in S^{1}$ be the $2 \pi$-periodic coordinate on $S^{1}$ and let $(x, y, z)$ be Euclidean coordinates on $\mathbb{R}^{3}$. Then

$$
\omega_{1}=2 z(d z \wedge d \alpha+d x \wedge d y)+x(d z \wedge d y-d x \wedge d \alpha)-y(d z \wedge d x+d y \wedge d \alpha)
$$

is a near-symplectic form on $S^{1} \times \mathbb{R}^{3}$ with vanishing locus $Z_{\omega_{1}}=S^{1} \times\{(0,0,0)\}$. Indeed, $\omega_{1}$ is self-dual with respect to $g=d z^{2}+d \alpha^{2}+d x^{2}+d y^{2}$ and transverse to the zero section of $\Lambda_{2}^{+}$. (Note that the symplectic orientation is opposite to the standard orientation on $S^{1} \times \mathbb{R}^{3}$.)

Letting $(r, \theta)$ be polar coordinates in the $(x, y)$-plane in $\mathbb{R}^{3}$, the form $\omega_{1}$ is invariant under the torus action $\left(t_{1}, t_{2}\right) \cdot(\alpha, r, \theta, z)=\left(\alpha+t_{1}, r, \theta+t_{2}, z\right)$. The orbit space can be identified with the closed upper half-plane $H=\{(X, Y) \mid Y \geq 0\}$, so that we have a singular fibration $\pi: S^{1} \times \mathbb{R}^{3} \rightarrow H$ whose fibers are orbits, which we may take to be given by the equations $X=z$ and $Y=r^{2} / 2$. This particular choice of parameterization facilitates certain calculations in the next section.

Note that if $(X, Y)$ is a point in the interior of $H$ then $\pi^{-1}(X, Y)$ is a torus orbit with trivial stabilizer, while if $(X, Y)$ lies on the $X$-axis then $\pi^{-1}(X, Y)$ is a circle orbit with stabilizer $G_{(1,0)}$.

While the half-plane $H$ is the orbit space, it is not the image of the moment map. To find the moment map, we find action-angle coordinates - coordinates on the union of principal orbits such that the moment map is projection to the linear coordinates. (Compare with Example 2.1) Accordingly, define $f: H \rightarrow \mathbb{R}^{2}$ by $p_{1}=X^{2}-Y, p_{2}=2 X Y$ 


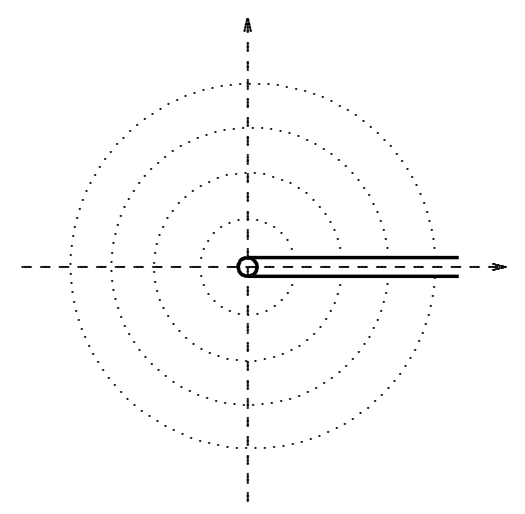

Fig. 2. The moment map image of the standard toric action near a component of the vanishing locus in a toric near-symplectic manifold.

and let $\mu_{1}=f \circ \pi: S^{1} \times \mathbb{R}^{3} \rightarrow \mathbb{R}^{2}$. Because $\mu_{1}$ is a submersion onto the complement of the positive $p_{1}$-axis and is torus invariant, we can pull $p_{1}, p_{2}$ back via $\mu_{1}$ and get coordinates

$$
p_{1}=z^{2}-r^{2} / 2, \quad q_{1}=\alpha, \quad p_{2}=z r^{2}, \quad q_{2}=\theta
$$

on the complement of $S^{1} \times \mathbb{R} \times\{0,0\}$, with respect to which $\omega_{1}=d p \wedge d q=d p_{1} \wedge$ $d q_{1}+d p_{2} \wedge d q_{2}$. The torus action then becomes $t \cdot(p, q)=(p, q+t)$ and the image of the union of circle orbits with stabilizer $G_{(1,0)}$ is the nonnegative $p_{1}$-axis. Consequently, as the reader can verify explicitly, $\mu_{1}$ is the moment map for the action on all of $S^{1} \times$ $\left(\mathbb{R}^{3} \backslash\{(0,0,0)\}\right)$, and hence a near-symplectic moment map on $S^{1} \times \mathbb{R}^{3}$.

The preimage under $\mu_{1}$ of a point on the positive $p_{1}$-axis is a disjoint union of two circle orbits and the preimage of the origin is one circle orbit, the one belonging to $Z_{\omega_{1}}$. To emphasize these features, we draw the moment map image as the $\left(p_{1}, p_{2}\right)$-plane with a double line along the positive $p_{1}$-axis and a hole at the origin; see Figure 2.

We now show that, up to an automorphism of the torus, these examples provide a complete set of examples.

First, it is important to understand the effect of an automorphism of the torus on the moment map. The following lemma is standard for symplectic manifolds (and is easily verified), and extends to near-symplectic manifolds by continuity.

Lemma 2.3. The moment map $\mu$ for a toric near-symplectic manifold $(X, \omega, \sigma)$ (where $\sigma$ is the torus action) is unique up to addition of a constant $b \in \mathbb{R}^{2}$. Furthermore, if $t \mapsto A t$, $A \in G L(2, \mathbb{Z})$, is any automorphism of the torus, then the toric manifold $\left(X, \omega, \sigma^{\prime}\right)$, with action $\sigma^{\prime}=\sigma \circ(A \times \mathrm{Id})$, has moment map $A^{T} \circ \mu$ where $A^{T}$ is the transpose of $A$.

Note that the set of orbit preserving symplectomorphisms is precisely the set of equivalence classes of equivariant symplectomorphisms in which two equivariant symplectomorphisms are deemed equivalent if one can be obtained from the other by precomposing with an automorphism of the torus. 
Lemma 2.4. Each orbit in a toric (symplectic) 4-manifold has a torus-invariant neighborhood that symplectically embeds, in an orbit-preserving fashion, into $\left(\mathbb{R}^{4}, \omega_{0}\right)$ equipped with the standard Hamiltonian torus action described in Example 2.1 Such an embedding can be chosen to be equivariant if and only if the stabilizer subgroup is the identity, $G_{(1,0)}, G_{(0,1)}$, or the whole torus.

Proof. The equivariant tubular neighborhood theorem (or slice theorem) states that any orbit has a neighborhood that is equivariantly symplectomorphic to a neighborhood of the zero section of its normal bundle equipped with a linear Hamiltonian torus action (cf. [2]). The germs of such equivariant neighborhoods are classified by their orbit types and their stabilizer subgroups. The standard Hamiltonian action on $\left(\mathbb{R}^{4}, \omega_{0}\right)$ has point and torus fibers, and circle orbits whose stabilizer subgroups are $G_{(1,0)}$ or $G_{(0,1)}$. The stabilizer subgroups for point and torus orbits are unique (equal to the torus and the identity, respectively). Meanwhile, an automorphism $A$ of the torus that is acting on the 4-manifold changes a stabilizer subgroup for a circle orbit from $G_{v}$ to $G_{A^{T} v}$, allowing any stabilizer subgroup to be achieved by an automorphism of the torus.

Observation 2.5. The moment map image of the neighborhood of a fixed point in a toric manifold is a convex sector bounded by rays with primitive integral tangent vectors $u, v$ such that the determinant $|u v|$ has norm 1, and conversely all such sectors appear as moment map images of $\mathbb{R}^{4}$. Meanwhile, the moment map image of the neighborhood of an orbit with stabilizer subgroup $G_{v}$ is a neighborhood of a point in a half-plane whose boundary has $v$ as its tangent vector.

Lemmas 2.4 and 2.3 tell us that Example 2.1 and its variants induced by automorphisms of the torus provide a complete set of local models for the neighborhood of an orbit in a toric symplectic manifold, and that these models are distinguished by their moment map images.

We now turn to the question of what toric structures can look like in the neighborhood of a component of the vanishing locus in a near-symplectic manifold.

Proposition 2.6. Each component $C$ of the vanishing locus in a toric near-symplectic manifold $(X, \omega, \sigma)$ has an open torus-invariant neighborhood $N$ and an orbit-preserving map $\phi:(N, \omega, \sigma) \rightarrow\left(S^{1} \times \mathbb{R}^{3}, \omega_{1}, \sigma_{1}\right)$ which is a smooth symplectic embedding of $N \backslash C$ that maps $C$ to $S^{1} \times 0$ and is a homeomorphism onto its image. Again, up to an automorphism of the torus, this embedding can be taken to be equivariant.

Proof. Whenever we use Cartesian coordinates $(x, y, z)$ on $\mathbb{R}^{3}$, then we will freely also use cylindrical coordinates $(r, \theta, z)$ on $\mathbb{R}^{3}$ without further ado.

Following the discussion of smooth torus actions in Section 2 (based on [16]), we know that, up to an automorphism of $T^{2}$, we can choose coordinates $\alpha \in S^{1},(x, y, z)$ $\in \mathbb{R}^{3}$ on a neighborhood $N$ of $C$ so that $C$ is $\{(\alpha, x, y, z) \mid x=y=z=0\}$ and so that the action is $\sigma\left(\left(t_{1}, t_{2}\right),(\alpha, r, \theta, z)\right)=\left(\alpha+t_{1}, r, \theta+t_{2}, z\right)$.

Suppose $\mu=\left(\mu_{1}, \mu_{2}\right): N \rightarrow \mathbb{R}^{2}$ is a moment map for $\sigma$ on $N$.

The orbit space is homeomorphic to the upper half-plane which, as in Example 2.2 we parameterize by coordinates $X, Y$ so that the projection $\pi$ to the orbit space is given 
by $X=z$ and $Y=r^{2} / 2$. (Note that we are using $(X, Y)$ as coordinates on this copy of $\mathbb{R}^{2}$ to distinguish them from $(x, y)$ which are coordinates on $N$.)

Then the moment map $\mu$ factors through the orbit space, $\mu=p \circ \pi$ where $p=$ $\left(p_{1}, p_{2}\right): H \rightarrow \mathbb{R}^{2}$. The map $p$ is smooth and is an immersion on $H \backslash(0,0)$. Because the isotropy subgroup of the circle orbits is $G_{(1,0)}$, the map $p$ sends both the positive and negative $X$-axes to straight lines of slope 0 . The fact that $\mu$ is a moment map for the given action then means that $\omega=d p_{1} \wedge d q_{1}+d p_{2} \wedge d q_{2}$, where $q_{1}=\alpha$ and $q_{2}=\theta$.

Since we can freely translate the image of a moment map and can apply the torus automorphism $\left(\begin{array}{cc}-1 & 0 \\ 0 & 1\end{array}\right)$ without changing the isotropy subgroup, we assume without loss of generality that $p$ maps the origin to the origin and the positive $X$-axis to the positive $p_{1}$-axis. Consider a small semicircular arc in $H$ starting on the positive $X$-axis and ending on the negative $X$-axis, avoiding $(0,0)$. This arc is mapped by $p$ to a path starting on the positive $p_{1}$-axis and ending either on the negative or positive $p_{1}$-axis, avoiding $(0,0)$, completing a total of $k$ half-rotations, for some positive integer $k$ which is independent of the choice of arc. We claim that $k=2$. In other words, $p$ also maps the negative $X$-axis to the positive $p_{1}$-axis and is injective on the interior of $H$.

We prove this claim by means of the following calculations.

We express $\omega$ in local coordinates near a point on $C$ as a map $\omega: \mathbb{R}^{4} \rightarrow \mathbb{R}^{6}$, where the $\mathbb{R}^{4}$-coordinates are $(\alpha, x, y, z)$ and the $\mathbb{R}^{6}$ coordinates are the coefficients of $(d \alpha \wedge d x, d \alpha \wedge d y, d \alpha \wedge d z, d x \wedge d y, d x \wedge d z, d y \wedge d z)$. We compute differentials:

$$
\begin{aligned}
& d p_{1}=x \frac{\partial p_{1}}{\partial Y} d x+y \frac{\partial p_{1}}{\partial Y} d y+\frac{\partial p_{1}}{\partial X} d z, \\
& d p_{2}=x \frac{\partial p_{2}}{\partial Y} d x+y \frac{\partial p_{2}}{\partial Y} d y+\frac{\partial p_{2}}{\partial X} d z, \\
& d q_{1}=d \alpha, \\
& d q_{2}=\frac{1}{r^{2}}(-y d x+x d y)=-\frac{y}{2} \frac{1}{Y} d x+\frac{x}{2} \frac{1}{Y} d y .
\end{aligned}
$$

Then because

$$
\begin{aligned}
d p_{1} \wedge d q_{1}+d p_{2} \wedge d q_{2}= & -x \frac{\partial p_{1}}{\partial Y} d \alpha \wedge d x-y \frac{\partial p_{1}}{\partial Y} d \alpha \wedge d y-\frac{\partial p_{1}}{\partial X} d \alpha \wedge d z \\
& +\frac{\partial p_{2}}{\partial Y} d x \wedge d y+\frac{y}{2}\left(\frac{1}{Y} \frac{\partial p_{2}}{\partial X}\right) d x \wedge d z \\
& -\frac{x}{2}\left(\frac{1}{Y} \frac{\partial p_{2}}{\partial X}\right) d y \wedge d z,
\end{aligned}
$$

we have the following expression for $\omega$ as a map to $\mathbb{R}^{6}$ :

$$
\omega(\alpha, x, y, z)=\left(-x \frac{\partial p_{1}}{\partial Y},-y \frac{\partial p_{1}}{\partial Y},-\frac{\partial p_{1}}{\partial X}, \frac{\partial p_{2}}{\partial Y}, \frac{y}{2}\left(\frac{1}{Y} \frac{\partial p_{2}}{\partial X}\right),-\frac{x}{2}\left(\frac{1}{Y} \frac{\partial p_{2}}{\partial X}\right)\right) .
$$

The derivative $D \omega$ is simply the 6-by-4 matrix of partial derivatives of this function. The claim will follow from the fact that, on the vanishing locus $Z_{\omega}=\{x=y=z=0\}$ where 
$X=Y=0$, this matrix must have rank 3 , together with the requirements that $\omega$ must be everywhere well-defined and equal to 0 on $Z_{\omega}$. For $\omega$ to be well-defined, $\partial p_{2} / \partial X$ must be divisible by $Y$, i.e.

$$
\frac{\partial p_{2}}{\partial X}=Y f(X, Y)
$$

for some smooth function $f$, and in particular

$$
\frac{\partial p_{2}}{\partial X}=0 \quad \text { when } \quad X=Y=0
$$

That $\omega$ must vanish on $Z_{\omega}$ implies

$$
\frac{\partial p_{1}}{\partial X}=\frac{\partial p_{2}}{\partial Y}=0 \quad \text { when } \quad X=Y=0 .
$$

Now we compute $D \omega$, noting that everything is $\alpha$-invariant, that for any function $h(X, Y)$ we have

$$
\frac{\partial h}{\partial x}=x \frac{\partial h}{\partial Y}, \quad \frac{\partial h}{\partial y}=y \frac{\partial h}{\partial Y}, \quad \frac{\partial h}{\partial z}=\frac{\partial h}{\partial X},
$$

and that

$$
\frac{\partial^{2} p_{2}}{\partial X \partial Y}=\frac{\partial}{\partial Y}(Y f(X, Y))=f(X, Y)+Y \frac{\partial f}{\partial Y}
$$

so that

$$
\frac{\partial^{2} p_{2}}{\partial X \partial Y}(0,0)=f(0,0)
$$

Thus, on $Z_{\omega}$ where $X=Y=0$, we get

$$
D \omega=\left[\begin{array}{cccc}
0 & -\frac{\partial p_{1}}{\partial Y} & 0 & 0 \\
0 & 0 & -\frac{\partial p_{1}}{\partial Y} & 0 \\
0 & 0 & 0 & -\frac{\partial^{2} p_{1}}{\partial X^{2}} \\
0 & 0 & 0 & f \\
0 & 0 & \frac{1}{2} f & 0 \\
0 & -\frac{1}{2} f & 0 & 0
\end{array}\right] .
$$

Therefore, to have rank 3 , we need either that

$$
f(0,0)=\frac{\partial^{2} p_{2}}{\partial X \partial Y}(0,0) \neq 0,
$$

or that

$$
\frac{\partial^{2} p_{1}}{\partial X^{2}}(0,0) \neq 0 \quad \text { and } \quad \frac{\partial p_{1}}{\partial Y}(0,0) \neq 0 .
$$

We now complete the proof of the claim by contradiction. Suppose $k \neq 2$. Note that $p_{2}$ is a real-valued function of two variables mapping $(0,0)$ to 0 with $(0,0)$ as a critical point. (Since $p_{2}$ is smooth on $H$ we may extend its domain to an open neighborhood of $H$.) If $k=1$ then $p_{2}(X, Y)>0$ for all $Y>0$, while $p_{2}(X, 0)=0$ for all $X$, so $(0,0)$ 
must be a degenerate critical point for $p_{2}$. Also if $k>2$ then $(0,0)$ must be a degenerate critical point, and so in either case the Hessian of $p_{2}$ at $(0,0)$ must be singular. Since $\frac{\partial p_{2}}{\partial X}=Y f(X, Y)$, we know that $\frac{\partial^{2} p_{2}}{\partial X^{2}}(0,0)=0$, so the Hessian being singular implies that at $(0,0)$ we have $\frac{\partial^{2} p_{2}}{\partial X \partial Y}=0$.

Now consider $p_{1}$. If $k=1$ then, along the $X$-axis, $p_{1}(X, 0)$ is an increasing function of $X$ whose first derivative vanishes at $X=0$, so its second derivative must also vanish at $X=0$, i.e. $\frac{\partial^{2} p_{1}}{\partial X^{2}}=0$ at $(0,0)$. On the other hand, if $k>2$, consider $p_{1}$ evaluated along semicircles centered at $(0,0)$ and of radius $\epsilon$, as $\epsilon \rightarrow 0$. Define $\Gamma_{\epsilon}:=\{(X, Y) \mid$ $X^{2}+Y^{2}=\epsilon^{2}$ and $\left.\frac{\partial^{2} p_{1}}{\partial X^{2}}=0\right\}$. On each semicircle, $\frac{\partial^{2} p_{1}}{\partial X^{2}}$ takes on both positive and negative values, so for each $\epsilon>0, \Gamma_{\epsilon}$ is nonempty. Since $\frac{\partial^{2} p_{1}}{\partial X^{2}}=0$ is a closed condition, $\bigcup_{\epsilon} \Gamma_{\epsilon}$ is a closed subset of $H$, implying that $\Gamma_{0}$ is nonempty. Therefore, $\frac{\partial^{2} p_{1}}{\partial X^{2}}=0$ at $(0,0)$.

Thus we must have $k=2$, which establishes the claim.

The above claim means that these coordinates $\left(p_{1}, q_{1}, p_{2}, q_{2}\right)$ behave, topologically, exactly the same as the $\left(p_{1}, q_{1}, p_{2}, q_{2}\right)$ coordinates in Example 2.2 Thus, identifying these coordinates here with the corresponding coordinates in Example 2.2 gives the desired homeomorphism which can fail to be smooth only on the vanishing locus $p_{1}=$ $p_{2}=0$.

\section{Toric near-symplectic manifolds}

Recall that a toric near-symplectic manifold is a near-symplectic manifold equipped with an effective smooth torus action that is Hamiltonian on the symplectic locus. As such, there are two relevant classifications that are well-understood: that of $T^{2}$-manifolds in the smooth category and that of toric manifolds in the symplectic category.

Orlik and Raymond classify $T^{2}$-manifolds in terms of their weighted orbit spaces. They first note that the orbit space is a surface with boundary such that each point in the interior of the surface is the image of a torus, and each point on the boundary is the image of a lower-dimensional fiber (circle or point). Furthermore, the boundary is a union of edges, the interiors of which parameterize circle orbits and the endpoints of which are the images of point orbits. The weighted orbit space of a $T^{2}$-manifold is then the oriented orbit space together with a labeling of the edges by the corresponding stabilizer subgroups.

Meanwhile, Delzant's Theorem classifies closed toric manifolds in terms of their moment map images [4], which are polygons (and in fact are weighted orbit spaces because the tangent vectors to their edges encode the stabilizer subgroups).

We cannot apply Delzant's classification to the symplectic locus of a toric nearsymplectic manifold because it is noncompact, thereby allowing the preimage of a point in the moment map image to be disconnected. To accommodate this feature we introduce integral affine surfaces, which are essentially weighted orbit spaces with an induced geometry that encodes the essential structure of the moment map. We then determine the extent to which one can classify such manifolds in terms of their orbit spaces equipped 
with integral affine structures (Theorem 3.7). For a more leisurely discussion of this matter in the symplectic case the reader can consult [18].

Consider a toric near-symplectic manifold $(X, \omega, \sigma)$ and the projection to its orbit space, $\pi: X \rightarrow B$. Let $F$ be the discrete subset of $\partial B$ that is the image of the vanishing locus. Then the restriction of the moment map $\mu$ to the symplectic locus factors through an orientation preserving immersion $\Phi: B \backslash F \rightarrow \mathbb{R}^{2}$ which extends to a continuous map $\bar{\Phi}$ such that $\mu=\bar{\Phi} \circ \pi$.

The immersion $\Phi$ induces a geometric structure on the orbit space whose local isometries are elements of $\operatorname{Aff}(2, \mathbb{Z})$. Henceforth, unless otherwise noted, we assume that "surface" means "surface with possibly nonempty boundary."

Definition 3.1. An integral affine structure on a surface is a maximal atlas of charts to a sector of $\mathbb{R}^{2}$ whose boundary rays have rational slope, with transition functions in $\operatorname{Aff}(2, \mathbb{Z})$. The standard integral affine structure on $\mathbb{R}^{2}$, denoted $\mathcal{A}_{0}$, is the atlas containing the identity map. Two integral affine surfaces $(B, \mathcal{A})$ and $\left(B^{\prime}, \mathcal{A}^{\prime}\right)$ are isomorphic if there exists a homeomorphism $\phi: B \rightarrow B^{\prime}$ such that $\phi^{*} \mathcal{A}^{\prime}=\mathcal{A}$.

Note that the homeomorphism $\phi$ is a diffeomorphism on the complement of the vertices.

Definition 3.2. A vertex on the boundary of an integral affine surface is a point that maps, via a chart, to a vertex of a sector in $\mathbb{R}^{2}$, while an edge is the closure of a connected component of the boundary minus its vertices. The boundary of an integral affine surface is right polygonal if every vertex has a neighborhood that is isomorphic to a neighborhood of the origin in the quadrant $\left(Q, \mathcal{A}_{0}\right) \subset\left(\mathbb{R}^{2}, \mathcal{A}_{0}\right)$.

For near-symplectic manifolds equipped with a toric structure, the local model near a component of the vanishing locus (Example 2.2) dictates the geometric structure in the base near the image of such a component (Figure 2). This inspires the following:

Definition 3.3. The boundary of an integral affine surface has edge folds if there is a set of points $F \subset \partial B$, called fold points, such that each point $p \in F$ has a neighborhood $U$ and a homeomorphism $\phi: U \rightarrow V$ to a neighborhood $V$ of $(0,0)$ in the upper half-plane $H=\{(X, Y) \mid Y \geq 0\}$, such that the integral affine structure on $U \backslash p$ is $\phi^{*} \psi^{*} \mathcal{A}_{0}$, where $\psi:(X, Y) \mapsto\left(X^{\overline{2}}-Y, 2 X Y\right)$ and $\mathcal{A}_{0}$ is the standard integral affine structure on $\mathbb{R}^{2}$.

If the set of fold points is nonempty, we always indicate these singularities explicitly, so the integral affine surface whose boundary has edge folds is a triple $(B, \mathcal{A}, F)$. Two surfaces with such structures, $(B, \mathcal{A}, F)$ and $\left(B^{\prime}, \mathcal{A}^{\prime}, F^{\prime}\right)$, are equivalent if there exists a homeomorphism from $B$ to $B^{\prime}$, taking $F$ bijectively to $F^{\prime}$, which is an integral affine equivalence of $(B \backslash F, \mathcal{A})$ and $\left(B^{\prime} \backslash F^{\prime}, \mathcal{A}^{\prime}\right)$.

The orbit space of a toric near-symplectic manifold acquires the structure of an integral affine surface whose boundary is right polygonal with edge folds by pulling back to $B \backslash F$ the standard integral affine structure via the immersion $\Phi$. Note that Lemma 2.3 implies that if two toric near-symplectic manifolds are equipped with actions that differ by an automorphism of the torus, then the integral affine structures on their orbit spaces are isomorphic. 
Definition 3.4. Given a toric near-symplectic manifold $(X, \omega, \sigma)$ with moment map $\mu$ and orbit space projection $\pi$, there is a unique map $\bar{\Phi}$ defined by $\mu=\bar{\Phi} \circ \pi$. The local models for the moment map near any orbit imply that $\bar{\Phi}$ is an immersion on the complement of a set of isolated points which we denote by $F$. Let $\Phi=\left.\bar{\Phi}\right|_{B \backslash F}$, where $B=$ $\pi(X)$. The integral affine base of the fibration defined by $\pi$ and $\mu$ is then $\left(B, \Phi^{*} \mathcal{A}_{0}, F\right)$, an integral affine surface whose boundary is right polygonal with edge folds.

Theorem 3.5. Suppose $(B, \mathcal{A}, F)$ is an integral affine surface whose boundary is right polygonal boundary with edge folds. Then $(B, \mathcal{A}, F)$ is the integral affine base of a toric near-symplectic manifold if and only if there is an integral affine immersion $\Phi$ : $(B \backslash F, \mathcal{A}) \rightarrow\left(\mathbb{R}^{2}, \mathcal{A}_{0}\right)$ that extends to a continuous map $\bar{\Phi}: B \rightarrow \mathbb{R}^{2}$ so that each point in $F$ has a neighborhood $N$ on which $\left.\bar{\Phi}\right|_{N \backslash \partial B}$ is injective and $\left.\bar{\Phi}\right|_{(N \backslash F) \cap \partial B}$ is two-to-one and linear.

Notice that there is no requirement here that $B$ be compact.

Proof. The "only if" direction follows directly from the factorization of the moment map for a toric near-symplectic manifold mentioned just before Definition 3.1 and the local models in Examples 2.1 and 2.2 together with Lemma 2.4 and Proposition 2.6 invoking automorphisms of the torus as needed. The "if" direction follows by construction.

Fix a torus $T^{2}$ with cyclic coordinates $q=\left(q_{1}, q_{2}\right)$ of period $2 \pi$ and consider the manifold $B \times T^{2}$ with the smooth torus action $t \cdot(x, q)=(x, q+t)$ where $t=\left(t_{1}, t_{2}\right)$. Equip $B \backslash F$ with local coordinates $\left(p_{1}, p_{2}\right)=\bar{\Phi}(x), x \in B$. Then $d p \wedge d q$ is a symplectic form on $(B \backslash F) \times T^{2}$ with respect to which the action is Hamiltonian. Notice that the moment map for this action amounts to forgetting the cyclic coordinates.

Take the quotient of $B \times T^{2}$ by identifying points as follows: if $x$ belongs to a vertex in the boundary of $(B, \mathcal{A}, F)$ then identify $(x, q)$ and $\left(x, q^{\prime}\right)$ for all $q, q^{\prime}$; if $x$ belongs to the interior of an edge $E$ of $B$, then identify $(x, q)$ and $\left(x, q^{\prime}\right)$ whenever $t \cdot(x, q)=\left(x, q^{\prime}\right)$ for some $t \in G_{v}$ where $v$ is a primitive integral tangent vector to $\bar{\Phi}(E)$. Example 2.1. together with automorphisms of the torus, and the fact that $(B \backslash F, \mathcal{A})$ is an integral affine surface with right polygonal boundary, ensure that the quotient is a smooth manifold with a smooth torus action, and that the symplectic structure (on the symplectic locus) descends to the quotient, making the action Hamiltonian there. Call this manifold $X$ and let $\omega$ be the symplectic structure defined on the symplectic locus. Let $\pi: X \rightarrow B$ be projection induced on $X$ by the projection $B \times T^{2} \rightarrow B$ that merely forgets the $T^{2}$ factor.

Now, for any point $p$ in $F$, let $N$ be a small neighborhood of $p$ satisfying the hypotheses. Let $N^{\prime}=N \backslash p$. Then by construction, up to an automorphism of the torus, $\left(\pi^{-1}\left(N^{\prime}\right),\left.\omega\right|_{\pi^{-1}\left(N^{\prime}\right)}\right)$ embeds symplectically and equivariantly into the complement of the vanishing locus in Example 2.2. Let $\phi$ be the continuous extension of this embedding to $\pi^{-1}(N)$. Pulling back via $\phi$ the near-symplectic structure $\omega_{1}$ and the torus action of Example 2.2 we get a toric structure on $\pi^{-1}(N)$ that is compatible with the toric structure on the symplectic locus of $X$. Doing this for each $p \in F$ completes the construction. 
The question of what integral affine surfaces immerse in $\left(\mathbb{R}^{2}, \mathcal{A}_{0}\right)$ is being investigated by the second author. However, there is one case in which one can be ensure of the existence of an integral affine immersion.

Lemma 3.6. If $(B, \mathcal{A})$ is an integral affine surface such that $B$ has trivial fundamental group, then there exists an integral affine immersion $(B, \mathcal{A}) \rightarrow\left(\mathbb{R}^{2}, \mathcal{A}_{0}\right)$.

Proof. $(B, \mathcal{A})$ is a manifold locally modeled on $\left(\mathbb{R}^{2}, \mathcal{A}_{0}\right)$. As such, its developing map (cf. [22]) $D:\left(\widetilde{B}, \Psi^{*} \mathcal{A}\right) \rightarrow\left(\mathbb{R}^{2}, \mathcal{A}_{0}\right)$ from the universal cover (whose covering map is $\left.\underset{\widetilde{B}}{\Psi}:\left(\widetilde{B}, \Psi^{*} \mathcal{A}\right) \rightarrow(B, \mathcal{A})\right)$ is an integral affine immersion. But since $B$ is contractible, $\widetilde{B}=B$ and $\Psi=$ Id.

We can now state and prove the generalization of Delzant's Theorem for toric near-symplectic manifolds.

Theorem 3.7. An integral affine surface $(B, \mathcal{A}, F)$, with possibly nonempty right polygonal boundary with edge folds, together with an integral affine immersion $\Phi:(B \backslash F, \mathcal{A})$ $\rightarrow\left(\mathbb{R}^{2}, \mathcal{A}_{0}\right)$, determines a toric near-symplectic manifold uniquely up to equivariant homeomorphisms that, restricted to the symplectic locus, are symplectomorphisms. The integral affine base $(B, \mathcal{A}, F)$ by itself determines the toric near-symplectic manifold up to an orbit-preserving homeomorphism that is a symplectomorphism on the symplectic locus.

Proof. Without loss of generality, we restrict to open covers such that each component of the vanishing locus belongs to a unique open set-so the gluing of open sets occurs only on the symplectic locus. Use Theorem 3.5 to construct a toric near-symplectic manifold $(X, \omega, \sigma)$ whose integral affine base is $(B, \mathcal{A}, F)$ with projection $\pi: X \rightarrow B$. Let $\mathcal{S}$ be the sheaf on $B$ that associates to any open set $U \subset B$ the group of equivariant symplectomorphisms of $\left(\pi^{-1}(U), \omega_{\pi^{-1}(U)}\right)$. The sheaf cohomology group $H^{1}(B, \mathcal{S})$ encodes the transition maps between such torus invariant neighborhoods and classifies, up to isomorphism, toric manifolds whose moment map $\mu$ factors as $\Phi \circ \pi$.

Our goal is to show that $H^{1}(B, \mathcal{S})$ is trivial. To do so we follow the argument put forth in [12, Prop. 7.3], which the reader may consult for further details. Since the equivariant symplectomorphisms are all time-one flows of Hamiltonian vector fields, $\mathcal{S}$ fits into an exact sequence of sheaves,

$$
0 \rightarrow \mathcal{L} \rightarrow \mathcal{C}^{\infty} \rightarrow \mathcal{S} \rightarrow 0
$$

where $\mathcal{C}^{\infty}$ is the sheaf of smooth torus invariant functions on $X$ (which is equivalent to the sheaf of smooth functions on $B)]^{1}$ and $\mathcal{L}$ is the sheaf of torus invariant functions whose time-one flows generate the identity map. This short exact sequence induces the long exact sequence

$$
\cdots \rightarrow H^{i}(B, \mathcal{L}) \rightarrow H^{i}\left(B, \mathcal{C}^{\infty}\right) \rightarrow H^{i}(B, \mathcal{S}) \rightarrow H^{i+1}(B, \mathcal{L}) \rightarrow \cdots,
$$

so it suffices to show that $H^{1}\left(B, \mathcal{C}^{\infty}\right)=H^{2}(B, \mathcal{L})=0$.

\footnotetext{
${ }^{1}$ In [12] the sheaf $\mathcal{C}^{\infty}$ is isomorphic to the sheaf of continuous functions on $B$ that lift to smooth functions on the total space because the authors are working with orbifolds.
} 
The sheaf $\mathcal{L}$ is isomorphic to the sheaf of locally constant functions on $B$ with values in $\mathbb{R} \times \Lambda^{*}$ where $\Lambda^{*} \cong \mathbb{Z}^{2}$ is the lattice in the Lie algebra $\mathbf{t}^{*}$ consisting of covectors $\xi$ whose infinitesimal vector field has the identity map as its time-one flow. Indeed, the elements of $\mathcal{L}$ are precisely the functions $f_{(c, \xi)}$ defined by $f_{(c, \xi)}(b)=c+\langle\xi, \Phi(b)\rangle$ for each $(c, \xi) \in \mathbb{R} \times \Lambda^{*}$. Because $B$ is locally contractible, we have isomorphisms between sheaf cohomology with coefficients in $\mathbb{R}$ and $\mathbb{Z}$, and both de Rham and singular cohomology. Consequently, $H^{2}(B, \mathcal{L})=0$ because

$$
H^{i}(B, \mathcal{L}) \cong H^{i}(B, \mathbb{R}) \times H^{i}(B, \mathbb{Z}) \times H^{i}(B, \mathbb{Z}),
$$

and the fact that $B$ immerses in $\mathbb{R}^{2}$ implies $H^{2}(B, \mathbb{Z}) \cong H^{2}(B, \mathbb{R})=0$. Meanwhile, because $\mathcal{C}^{\infty}$ is a fine sheaf, $H^{i}\left(B, \mathcal{C}^{\infty}\right)=0$ for all $i>0$.

A different choice of integral affine immersion would yield a new toric manifold with moment map $\Phi^{\prime}=\Psi \circ \Phi$ for some $\Psi \in \operatorname{Aff}(2, \mathbb{Z})$. If $A$ were the linear part of $\Psi$, then by Lemma 2.3 and the uniqueness proved above, the corresponding toric manifold would be $\left(X, \omega, \sigma^{\prime}\right)$ with $\sigma^{\prime}=\sigma \circ\left(A^{T} \times \mathrm{Id}\right)$, which is indeed orbit-preserving symplectomorphic to $(X, \omega, \sigma)$.

The fact that our maps may not be smooth across the vanishing loci is a reflection of the fact [9] that Moser's method for near-symplectic forms near their vanishing loci does not give smoothness at the vanishing loci. Thus it is really a feature of the germ of the toric structure on a component of the vanishing locus, rather than a feature of how we glue in a neighborhood of the component.

\section{Making $T^{2}$-manifolds near-symplectic}

\subsection{Simply connected $T^{2}$-manifolds}

Equipped with Orlik and Raymond's technology [16] for understanding torus actions in the smooth category, as outlined in Section 3, we now investigate the problem of finding near-symplectic structures adapted to given smooth torus actions. The goal is to prove Theorems 1.10, 1.12 and Proposition 1.11 In light of Theorem 3.7 proving Theorems 1.10 and 1.12 amounts to realizing given weighted orbit spaces as integral affine surfaces with appropriate boundaries isometrically immersed in $\left(\mathbb{R}^{2}, \mathcal{A}_{0}\right)$.

We separate Theorem 1.10 into three propositions $4.1,4.10$, and 4.11 , the first of which is:

Proposition 4.1. Every locally toric fibration of a closed simply connected near-symplectic manifold is toric.

Proof. Let $\pi:(X, \omega) \rightarrow(B, \mathcal{A}, F)$ be the locally toric fibration. By Theorem 3.5. $(X, \omega)$ is toric if there is an integral affine immersion $\Phi:(B \backslash F, \mathcal{A}) \rightarrow\left(\mathbb{R}^{2}, \mathcal{A}_{0}\right)$.

Because any loop in $B$ lifts to a loop in $X, X$ being simply connected implies $B$ is simply connected. Because a sphere cannot admit an integral affine structure, $B$ must be homeomorphic to a disk. Finally, by Lemma 3.6 we know that $(B \backslash F, \mathcal{A})$ does immerse isometrically in $\left(\mathbb{R}^{2}, \mathcal{A}_{0}\right)$. 
Before proving the rest of Theorem 1.10 we introduce language to describe the image of the boundary of an immersed integral affine surface.

Definition 4.2. A list of slopes $s_{1}, \ldots, s_{k}$, or slope list, is right polygonal if $s_{j} \in \mathbb{Q} \cup \infty$ for each $j$ and, writing $s_{j}=m_{j} / n_{j}$ as a reduced fraction (with $m_{j}=1, n_{j}=0$ if $\left.s_{j}=\infty\right)$, if the determinant $\left(\begin{array}{cc}m_{j} & m_{j+1} \\ n_{j} & n_{j+1}\end{array}\right)$ has norm 1 for each $j \leq n-1$. When the indices of a list of $k$ slopes are understood mod $k$, the slope list is cyclic.

Definition 4.3. A polygonal path with folds is a piecewise linear map $\gamma:[0, n] \rightarrow$ $\mathbb{R}^{2}, n \in \mathbb{N}$, well defined up to reparameterization relative endpoints on the subintervals $[j-1, j], j=1, \ldots, N$, such that

(1) the image $e_{j}$ of $\left.\gamma\right|_{[j-1, j]}, j \in\{1, \ldots, N\}$, has constant slope (and is called an edge),

(2) the slopes of $e_{j}$ and $e_{j+1}$ are different for each $j=1, \ldots, N-1$.

The vertices are the images of the integral points, $\gamma(j)$, while the fold points are the interior points of edges at which $\gamma$ is not smooth, i.e. where the oriented tangent vector changes direction.

A polygonal path (possibly with folds) is right polygonal if each vertex $\gamma(j)$ has a neighborhood that contains no fold points and in which oriented tangent vectors $v_{j}$ and $v_{j+1}$ to the edges $e_{j}, e_{j+1}$ have determinant $\left|v_{j} v_{j+1}\right|=1$.

The definitions are analogous for polygonal loops with the usual additional stipulation that $\gamma(0)=\gamma(n)$. Notice that polygonal paths and loops are oriented in accordance with the standard orientation of the domain interval $[0, n]$ - and the indexing of the edges and vertices.

Given a (cyclic) slope list, we say that a polygonal path (or loop) with folds realizes this list if the list of slopes of the edges, ordered according to the orientation, equals the given slope list.

Definition 4.4. Abusing correct terminology considerably, let us say that a polygonal path with folds is embedded if the only intersections between distinct edges occur when two consecutive edges meet at a vertex and if there are no triple points.

In other words, an edge with a fold obviously intersects itself, but this is the only type of self-intersection allowed and such an edge may not intersect itself too much. In particular a given edge may have at most two folds. To justify the use of the term "embedded", note that if we remove all double points and take the closure of the remainder, we get an honestly embedded polygonal path.

Definition 4.5. Given two slopes $s_{1}, s_{2} \in \mathbb{Q} \cup\{\infty\}$, define the positive angle from $s_{1}$ to $s_{2}$ to be the angle $\alpha\left(s_{1}, s_{2}\right) \in[0, \pi)$ from a line of slope $s_{1}$ to a line of slope $s_{2}$ measured counterclockwise. Given a list of slopes $s_{1}, \ldots, s_{n} \in \mathbb{Q} \cup\{\infty\}$, define the positive turning for the list to be $T:=\alpha\left(s_{1}, s_{2}\right)+\cdots+\alpha\left(s_{n-1}, s_{n}\right)$ or, if the list is cyclic, $T:=\alpha\left(s_{1}, s_{2}\right)+$ $\cdots+\alpha\left(s_{n-1}, s_{n}\right)+\alpha\left(s_{n}, s_{1}\right)$. Given a $T^{2}$-manifold with weighted orbit space $B$, the positive turning for a component of $\partial B$ with weights $\left\{m_{i} / n_{i}\right\}, i=1, \ldots, n$, is the positive turning for the cyclic slope list $\left\{m_{i} / n_{i}\right\}, i=1, \ldots, n$. 
Note that, for any list, $T=k \pi$ for some nonnegative integer $k$, and that $T=0$ if and only if the boundary component has only one edge.

Definition 4.6. Given a polygonal path with folds, define the total turning to be the sum of the amounts of counterclockwise turning of the tangent vectors at the vertices (between 0 and $\pi$ ) and at the folds (always $-\pi$ ). If the path is closed, we include the turning at the initial vertex (which is also the final vertex).

The following is an immediate consequence of Definitions 4.4, 4.5 and 4.6 .

Lemma 4.7. Given a slope list with positive turning $T$ and a right polygonal loop with folds realizing the list with total turning $T^{\prime}$, we have $T^{\prime}=T-|F| \pi$, where $|F|$ is the number of folds. An embedded right polygonal loop with folds in $\mathbb{R}^{2}$ always has total turning $\pm 2 \pi$.

Lemma 4.8. Given a right polygonal slope list $s_{1}, \ldots, s_{k}, k \geq 2$, with $s_{1}=0$ and $s_{2}=\infty$, and a point $\left(x_{0}, y_{0}\right) \in \mathbb{R}^{2}$ with $x_{0}, y_{0}>0$, there exists an embedded right polygonal path with folds that realizes the slope list, has initial endpoint $(0,0)$ and final endpoint $\left(x_{0}, y_{0}\right)$, and whose first edge $e_{1}$ has oriented tangent vector $(1,0)$.

Proof. Let $a, b, \delta$ and $\epsilon$ be indeterminates that will be fixed later. Let $e_{1}=[0, a] \times\{0\}$ and let $e_{2}$ be an edge that starts with $\{a\} \times[0, b]$, has a fold at $(a, b)$ and then doubles back a distance $\delta$, ending at $(a, b-\delta)$. Now turn counterclockwise onto an edge $e_{3}$ of slope $s_{3}$ and length $\epsilon$. Henceforth, turn counterclockwise from $e_{i}$ onto an edge $e_{i+1}$ of slope $s_{i+1}$ and length $\epsilon$ if this does not require moving in the negative $x$ direction. Otherwise, put a fold at the current end of $e_{i}$ and extend $e_{i}$ by doubling back a distance $\delta$, then turn counterclockwise onto $e_{i+1}$, of slope $s_{i+1}$ and length $\epsilon$. Continue up to $e_{k}$. Given any preassigned $\lambda>0$, there exists a choice of $\epsilon$ and $\delta$ ( $\epsilon$ small and $\delta$ much smaller $)$ such that, for any $a, b>0$, the entire path is embedded and ends at $\left(a+a_{0}, b+b_{0}\right)$, where $0 \leq a_{0}<\lambda$ and $-\lambda<b_{0}<\lambda$. Then, if we choose $a=x_{0}-a_{0}$ and $b=y_{0}-b_{0}$, we can arrange that the path ends at $\left(x_{0}, y_{0}\right)$.

Notice that a slope list $s_{1}, \ldots, s_{n}$ can be realized by a right polygonal path if and only if one can also realize the slope list $s_{1}^{\prime}, \ldots, s_{n}^{\prime}$ where the reduced fractions $m_{i} / n_{i}$ and $m_{i}^{\prime} / n_{i}^{\prime}$ representing the slopes satisfy $\left(n_{i}^{\prime}, m_{i}^{\prime}\right)^{T}=A\left(n_{i}, m_{i}\right)^{T}$ for some fixed $A \in G L(2, \mathbb{Z})$.

Lemma 4.9. Any cyclic reduced slope list with positive turning $T \geq 2 \pi$ can be realized by an embedded right polygonal loop with folds.

Proof. Let $s_{1}, \ldots, s_{n}$ be the given list. Without loss of generality assume that $s_{1}=0$ and, for each $i, s_{i} \neq \infty$. Let $p$ be the smallest integer between 2 and $n$ such that $s_{p} \neq 0$ and such that the cyclic slope list $s_{1}, s_{p}, \ldots, s_{n}$ has total positive turning $2 \pi$. (Notice that this new slope list may fail to be right polygonal at the intersection of the $p^{\text {th }}$ and $1^{\text {st }}$ edges.) Without loss of generality we can also assume that $s_{p}>0$. If $p=2$ then it is standard to construct the desired loop, a closed convex polygon without folds. Now assume $p>2$.

Figure 3 illustrates the following construction. 


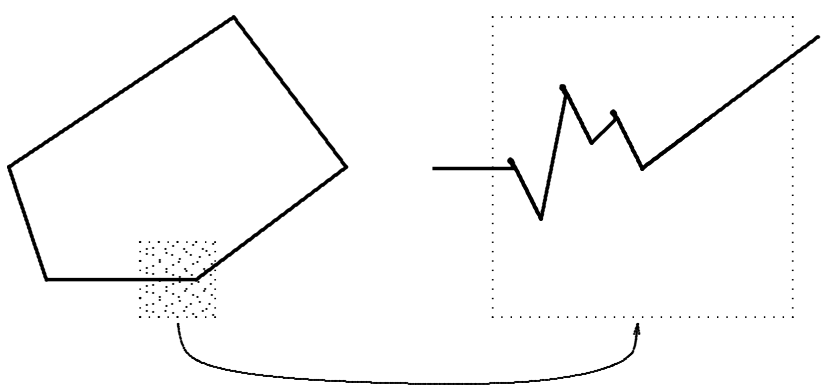

Fig. 3. An example construction of a right polygonal loop with folds.

First construct a closed convex polygon (without folds) with edges $e_{1}, e_{p}, \ldots, e_{n}$ realizing the cyclic slope list $s_{1}, s_{p}, \ldots, s_{n}$, with the vertex joining $e_{1}$ to $e_{p}$ located at $(0,0)$. Next, choose some $\left(x_{0}, y_{0}\right)$ on the interior of $e_{p}$ and replace the portion of $e_{p}$ connecting $(0,0)$ and $\left(x_{0}, y_{0}\right)$ by a right polygonal path representing the slope list $s_{1}, \ldots, s_{p-1}$, constructed as in Lemma 4.8. Note that in doing so the edge $e_{p}$ is shortened, its initial point becoming $\left(x_{0}, y_{0}\right)$, while $e_{1}$ is lengthened, its final point becoming $(a, 0)$ rather than $(0,0)$. We can ensure that this procedure yields an embedded polygonal loop by choosing the point $\left(x_{0}, y_{0}\right)$ sufficiently close to $(0,0)$ and the parameter $\lambda$ of Lemma 4.8 sufficiently small.

The next part of Theorem 1.10 that we prove is:

Proposition 4.10. Every closed simply connected $T^{2}$-manifold $(X, \sigma)$ with $b_{2}^{+}(X)>0$ is toric with respect to some near-symplectic structure.

Proof. The $T^{2}$ structure yields a slope list with positive turning $T$ for some $T \geq 0$. Assume for the moment that $T \geq 2 \pi$.

Appealing to Lemma 4.9 and its proof, construct an embedded right polygonal loop with folds representing the given slope list, thereby defining a near-symplectic structure with respect to which $(X, \sigma)$ is toric. This loop is then the right polygonal boundary with edge folds of an integral affine disk.

If $T \leq \pi$ any attempt to draw such an embedded polygonal loop fails: all right polygonal paths representing the slope list fail to close up. It remains to show that $T \leq \pi$ cannot occur. First note the $T=0$ cannot occur for any $T^{2}$-manifold because a slope list with just one slope would define a weighted orbit space with just one edge and one vertex. With only one isotropy subgroup, it is impossible to satisfy the condition that $|u v|= \pm 1$ where $G_{u}, G_{v}$ are the isotropy subgroups of circle orbits in the neighborhood of the fixed point.

Now suppose $T=\pi$. Consider the weighted orbit space for $(X, \sigma)$ with edges $e_{1}, \ldots, e_{k}$ having slopes $s_{i}=m_{i} / n_{i}$ for each $i=1, \ldots, k$, where the signs of $m_{i}, n_{i}$ are chosen so that $\left|\begin{array}{cc}n_{i} & n_{i+1} \\ m_{i} & m_{i+1}\end{array}\right|=1$ for each $i=1, \ldots, k-1$. (The fact that $T=\pi$ corresponds to the fact that, with these choices of signs, $\left|\begin{array}{ll}n_{k} & n_{1} \\ m_{k} & m_{1}\end{array}\right|=-1$.) 
Consider the $T^{2}$-manifold defined by the weighted orbit space that is a disk with weights given by the cyclic slope list $s_{1}, \ldots, s_{k}, s_{k+1}, s_{k+2}$ where $s_{k+1}=s_{1}$ and $s_{k+2}=m_{k+2} / n_{k+2}$ with $m_{k+2}, n_{k+2}$ satisfying $\left(\begin{array}{cc}n_{k+2} & n_{1} \\ m_{k+2} & m_{1}\end{array}\right)=1$. Call this new $T^{2}$ manifold $\left(X^{\prime}, \sigma^{\prime}\right)$. Then, topologically, $X^{\prime}$ is obtained from $X$ by removing a copy of $S^{1} \times D^{3}$ and gluing in a $D^{2} \times S^{2}$. Therefore $b_{2}^{+}\left(X^{\prime}\right)=b_{2}^{+}(X)+1$.

Because

$$
\left|\begin{array}{cc}
n_{k} & -n_{k+1} \\
m_{k} & -m_{k+1}
\end{array}\right|=\left|\begin{array}{ll}
-n_{k+1} & n_{k+2} \\
-m_{k+1} & m_{k+2}
\end{array}\right|=\left|\begin{array}{cc}
n_{k+2} & n_{1} \\
m_{k+2} & m_{1}
\end{array}\right|=1,
$$

the total turning of the new cyclic slope list is $2 \pi$ and $X^{\prime}$ admits a symplectic structure for which $\sigma^{\prime}$ is Hamiltonian. Therefore $b_{2}^{+}\left(X^{\prime}\right)=1$, implying $b_{2}^{+}(X)=0$.

Finally, the third part of Theorem 1.10 is:

Proposition 4.11. If $(X, \omega, \sigma)$ is a closed toric near-symplectic manifold that is simply connected, then the vanishing locus $Z_{\omega}$ must have exactly $\left|Z_{\omega}\right|=b_{2}^{+}(X)-1$ components.

Notice that Proposition 4.11 is trivially true when $\left|Z_{\omega}\right|=0$ because the only toric symplectic manifolds are $S^{2} \times S^{2}$ and $\mathbb{C} P^{2} \# \overline{\mathbb{C} P}^{2}$, all of which have $b_{2}^{+}=1$.

Example 4.12. Consider the moment map image of $\mathbb{C} P^{2} \# \mathbb{C} P^{2}$ shown in Figure 1 , for which one can easily verify Proposition 4.11. This toric near-symplectic manifold is an equivariant connected sum, and the decomposition can be performed via a symplectic cut [11] along the preimage of the vertical line segment that connects the fold point and the horizontal edge (cutting $X$ along that 3-sphere and then collapsing the circles on the resulting boundaries that are in the kernel of the symplectic form). Note that the preimage of the vertical segment is indeed a 3-sphere because the circle orbits mapping to the endpoints of the segment have isotropy subgroups $G_{(1,0)}$ and $G_{(0,1)}$, and $\left|\begin{array}{ll}1 & 0 \\ 0 & 1\end{array}\right|$ has modulus 1 .

The essence of our proof of Proposition 4.11 is to decompose $(X, \omega)$ via symplectic cutting as a connected sum of manifolds, each of which has no vanishing locus, and show that the quantity $b_{2}^{+}(X)-\left|Z_{\omega}\right|$ remains constant for all the manifolds involved. However, in general a toric manifold may have a 3 -sphere on which a one-dimensional subtorus acts freely, with one orbit being a component of the vanishing locus, i.e. a 3-sphere whose moment map image is a line segment with one endpoint being a fold point. For instance, consider the $T^{2}$-manifold with weighted orbit space whose slope list is $\infty, 0,-1,-2$. This manifold is diffeomorphic to $\mathbb{C} P^{2} \# \mathbb{C} P^{2}$, but is not an equivariant connected sum. (We leave it to the reader to use the techniques of this section to draw the moment map image for this action.) Here we thank Brett Parker for asking us a question which brought the possibility of such examples to our attention.

We circumvent this by recognizing that

(1) in light of Lemma $4.7, b_{2}^{+}(X)-\left|Z_{\omega}\right|$ depends only on the underlying $T^{2}$-manifold and the existence of a near-symplectic structure that makes the $T^{2}$-manifold toric, but does not depend on the particular near-symplectic structure;

(2) the quantity $b_{2}^{+}(X)-\left|Z_{\omega}\right|$ is invariant under equivariant blow-ups. 
Proof of Proposition 4.11. In this proof, let manifolds have more than one connected component. Let $\left(X_{i}, \omega_{i}, \sigma_{i}\right), i=1, \ldots, N$, be a sequence of toric near-symplectic manifolds such that $\left(X_{1}, \omega_{1}, \sigma_{1}\right)$ and $(X, \omega, \sigma)$ are equivalent as $T^{2}$-manifolds, $\left|Z_{i+1}\right|=$ $\left|Z_{i}\right|-1,\left|Z_{n}\right|=0$, and $\left(X_{i+1}, \omega_{i+1}, \sigma_{i+1}\right)$ is obtained from $\left(X_{i}, \omega_{i}, \sigma_{i}\right)$ by

(1) choosing a convenient near-symplectic structure on the $T^{2}$-manifold $\left(X_{i}, \sigma_{i}\right)$,

(2) performing equivariant blow-ups of some connected component of $\left(X_{i}, \sigma_{i}\right)$ as necessary, and then

(3) performing a symplectic cut of that component along a 3-sphere that contains a component of the vanishing locus.

The proposition will be proved if we show that $b_{2}^{+}\left(X_{i}\right)-\left|Z_{i}\right|=c_{i}$ where $c_{i}$ is the number of connected components of $X_{i}$. This is true for $X_{N}$ because $\left|Z_{N}\right|=0$ and each component has $b_{2}^{+}=1$. All we need to do is show that we can always find a nearsymplectic structure such that the toric manifold with underlying $T^{2}$-manifold $\left(X_{i}, \sigma_{i}\right)$, or a blow-up of it, can be equivariantly decomposed so as to reduce by 1 the number of fold points in the boundary of its moment map image.

Given the toric manifold $\left(X_{i}, \omega_{i}, \sigma_{i}\right)$, choose a connected component that contains a component of the vanishing locus and construct its moment map image as in the proof of Lemma 4.9. Recall that, by construction, the edge $e_{2}$ is vertical and contains one fold point. If the vertical line segment on the interior of the image with one endpoint at the fold point of $e_{2}$ has its other vertex on an edge of integral slope $m_{i} \in \mathbb{N}$, then perform a symplectic cut, eliminating the fold point on edge $e_{2}$. As in Example 4.12 this is possible because the determinant $\left|\begin{array}{ll}0 & 1 \\ 1 & m_{j}\end{array}\right|$ has modulus 1 .

If not, proceed as follows, noting that on edges $e_{p}, \ldots, e_{n}$ there are no fold points.

(1) Suppose there is an edge $e_{j}, j \geq p$, such that

(a) $e_{j}$ has integral slope $m_{j} \neq 0$,

(b) $e_{j}$ lies in a closed half-plane whose boundary contains the edge $e_{2}$,

(c) the lower vertex of $e_{j}$ is higher than the fold point $p$.

Then lengthen $e_{j}$ and $e_{3}$ (or $e_{1}$, depending on which is on the opposite side of $l$ from $e_{j}$ ), scaling the edges $e_{4}, \ldots, e_{j-1}$ (or $e_{j+1}, \ldots, e_{n}$ ) by a single constant so as to maintain an embedded polygonal path with folds.

(2) Or, if there is an edge $e_{j}, j \geq p$, that has slope 0 and lies above $p$, then lengthen $e_{j+1}$ and $e_{3}$ (or $e_{j-1}$ and $e_{1}$, depending on which side of $e_{2}$ the edge $e_{j}$ lies on), scaling the edges $e_{4}, \ldots, e_{j-1}$ (or $e_{j+1}, \ldots, e_{n}$ ) by a single constant so as to maintain an embedded polygonal path with folds.

(3) Or, if there are no edges with integral slope, find the vertex with maximal $y$ coordinate and name the edges on its left and right $e_{j}$ and $e_{j-1}$ with slopes $s_{j}=m_{j} / n_{j}$ and $s_{j-1}=m_{j-1} / n_{j-1}$. Remove a neighborhood of the corner, shortening $e_{j}$ and $e_{j-1}$ and inserting a new edge $e^{\prime}$ whose slope equals zero. If $m_{j}=1\left(m_{j-1}=1\right)$ then the boundary of the polygon is right polygonal at the left (right) vertex of $e^{\prime}$. If not, the vertex defines an orbifold singularity. But any such singularity can be resolved equivariantly, replacing the singular point with a union of spheres. After resolving, the polygon will have right polygonal boundary. Next, go back to Step 2 . 
Now we may perform a symplectic cut to obtain $\left(X_{i+1}, \omega_{i+1}, \sigma_{i+1}\right)$. If we carry out this procedure $N=|Z|$ times, we obtain $\left(X_{N}, \omega_{N}, \sigma_{N}\right)$ for which $b_{2}^{+}\left(X_{N}\right)-\left|Z_{N}\right|=c_{N}$. Since this relation remains unchanged through all of our constructions and $X$ is connected, $b_{2}^{+}\left(X_{1}\right)-\left|Z_{1}\right|=b_{2}^{+}(X)-\left|Z_{\omega}\right|=1$.

Proposition 1.11 allows us to recognize a simply connected $T^{2}$-manifold from its weighted orbit space, provided the orbit space has at least five vertices. An ingredient in its proof is the calculation of the Euler characteristic of a locally toric near-symplectic manifold from its weighted orbit space.

Lemma 4.13. Given a locally toric near-symplectic manfiold $(X, \omega, \sigma)$ with integral affine base $(B, \mathcal{A}, F), \chi(X)=V$ where $V$ is the number of vertices on the boundary of the integral affine base.

Recall that fold points do not count as vertices.

Proof. The total space of a locally toric manifold can be built up out of open sets, each of which is a neighborhood of a fiber. This can be done so that each neighborhood has Euler characteristic equal to 0 , except for a small neighborhood of the preimage of each vertex (which can be chosen to be a ball with Euler characteristic equal to 1). Furthermore, one can perform this operation so that as each neighborhood gets glued in, the gluing locus has Euler characteristic equal to 0 .

Proof of Proposition 1.11. If the orbit space has at least five vertices then it must be diffeomorphic to a connected sum of copies of $\mathbb{C} P^{2}$ and $\overline{\mathbb{C P}}^{2}$ (cf. [16]). Then, invoking Proposition 4.11 and Lemma 4.7, we can calculate

$$
m=b_{2}^{+}(X)=\left|Z_{\omega}\right|+1=|F|+1=\frac{T-2 \pi}{\pi}+1=\frac{T}{\pi}-1 .
$$

Then

$$
n=b_{2}^{-}(X)=\chi(X)-b_{2}^{+}(X)-2=V-m-2,
$$

where the last equality follows from Lemma 4.13

\section{2. $T^{2}$-manifolds with nontrivial fundamental groups}

The orbit space of any $T^{2}$-manifold whose fundamental group is nontrivial must also have nontrivial fundamental group. In order to describe and construct integral affine structures on orbit spaces of closed manifolds we define a few noncompact integral affine surfaces that serve as building blocks.

Definition 4.14. Given an open interval $I$ in the positive reals, let $A_{I}$ be the annulus $A_{I}=\left\{(x, y) \mid x^{2}+y^{2} \in I\right\}$. For any positive integer $q$, define the $q$-fold integral affine structure $\mathcal{A}_{I, q}$ on $A_{I}$ to be the pullback of the standard integral affine structure on $A_{I} \subset \mathbb{R}^{2}$ via the $q$-fold cover $(r, \theta) \mapsto(r, q \theta)$. 

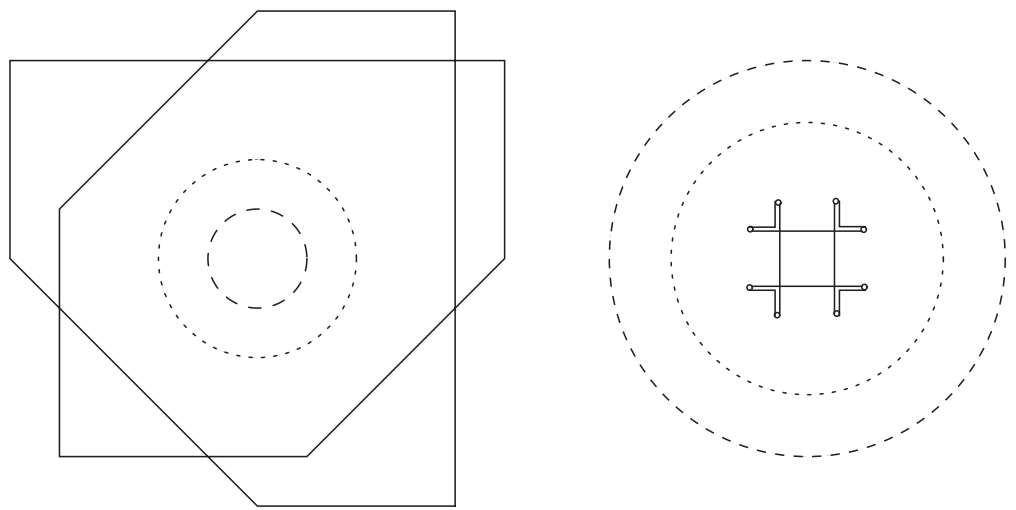

Fig. 4. On the left, an integral affine annulus with a 2-hole, and on the right, a 3-plug homeomorphic to an annulus. (In fact, the figure on the right could also represent a 1-plug homeomorphic to a twice-punctured torus.)

Definition 4.15. An integral affine surface with right polygonal boundary and edge folds $(B, \mathcal{A}, F)$ is a q-plug if $B$ has exactly one end modeled on the outer end of $A_{(a, b)}$ (for some interval $(a, b) \subset \mathbb{R})$, i.e. if there is an integral affine embedding $\Phi:\left(A_{(a, b)}, \mathcal{A}_{q}\right) \rightarrow$ $(B \backslash F, \mathcal{A})$ such that $B_{0}:=B \backslash \Phi\left(A_{(a, b)}\right)$ is compact and any sequence of points in $A_{(a, b)}$ converging to $r=a$ is sent via $\Phi$ to a sequence of points converging to $\partial B_{0} \subset B$.

Definition 4.16. An integral affine surface with right polygonal boundary and edge folds $(B, \mathcal{A}, F)$ has a $q$-hole if $B$ has one end modeled on the inner end of $A_{(a, b)}$ (for some interval $(a, b) \subset \mathbb{R})$, i.e. if there is an integral affine embedding $\Phi:\left(A_{(a, b)}, \mathcal{A}_{q}\right) \rightarrow$ $(B \backslash F, \mathcal{A})$ such that $B_{0}:=B \backslash \Phi\left(A_{(a, b)}\right)$ is connected and any sequence of points in $A_{(a, b)}$ converging to $r=b$ is sent via $\Phi$ to a sequence of points converging to $\partial B_{0} \subset B$.

Figure 4 shows the immersed images of an integral affine annulus with a 2-hole (without any folds) and a 3-plug (with four folds). The shaded regions are the annular ends.

Lemma 4.17. Given any $q$ and any cyclic slope list $s_{1}, \ldots, s_{n}$ that is right polygonal, there exists a q-plug whose boundary realizes the given slope list.

Proof. Without loss of generality, assume $s_{1}=0$ and add a slope, forming the slope list $s_{1}, s_{2}, \ldots, s_{n}, s_{n+1}$ with $s_{1}=s_{n+1}=0$. Use Lemma 4.8 to construct a right polygonal path ending at $\left(x_{0}, y_{0}\right)$ and representing this new slope list $s_{1}, s_{2}, \ldots, s_{n}, s_{n+1}$. By construction, $\left(x_{0}, y_{0}\right)$ is in the interior of the first quadrant. Extend $e_{2}$ near the endpoint it shares with $e_{3}$ by the length $y_{0}$, thereby "lowering" the part of the path representing the slopes $s_{3}, \ldots, s_{n}, s_{n+1}$. The result is a right polygonal path with endpoints at $(0,0)$ and $\left(x_{0}, 0\right)$. Replace these two endpoints with fold points, concatenating the edges $e_{n+1}$ and $e_{1}$. Although the resulting path is probably no longer embedded, it can be taken as the restriction to one boundary component of a continuous map of a closed annulus into $\mathbb{R}^{2}$. Furthermore, this continuous map can be assumed to be an immersion on the complement of a finite number of points on that boundary component and to map the other 


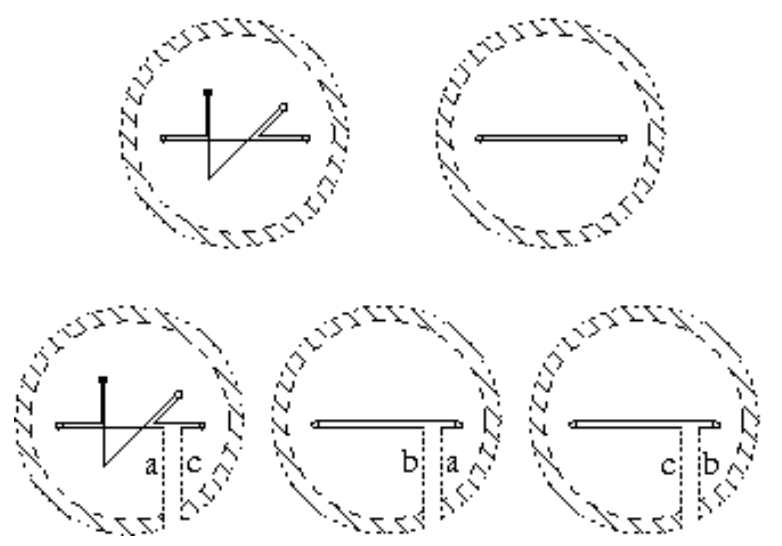

Fig. 5. Constructing a plug: Top left, a 1-plug with three edges. Top right, a 1-plug with one edge. On the bottom row, we indicate how to cut these open and glue them together (one of the first and two of the second) to make a 3-plug with three edges (letters $a, b, c$ indicate gluing instructions).

boundary component to a large circle. Then the interior of this annulus, with the integral affine structure induced by the immersion into $\left(\mathbb{R}^{2}, \mathcal{A}_{0}\right)$, is a 1-plug. To get a $q$-plug for $q>1$, introduce $2(q-1)$ more folds in the edge $e_{1}$; see Figure 5

We now prove Theorem 1.12 which asserts that there exists a near-symplectic structure making a torus action Hamiltonian only if there is a component of the boundary of the weighted orbit space on which the positive turning is at least $2 \pi$; that this suffices if the genus of the base is zero; and that a compact surface of any given genus and at least one boundary component can be the orbit space for a toric near-symplectic manifold.

Proof of Theorem 1.12 Suppose $(X, \sigma)$ admits a near-symplectic structure with respect to which the action is Hamiltonian. Let $(B, \mathcal{A}, F)$ be the integral affine base and $\bar{\Phi}$ : $B \rightarrow \mathbb{R}^{2}$ be the continuous extension of the integral affine immersion $\Phi:(B \backslash F, \mathcal{A}) \rightarrow$ $\left(\mathbb{R}^{2}, \mathcal{A}_{0}\right)$ that defines the action on the symplectic locus. The image under $\bar{\Phi}$ of each component of $\partial B$ is the image of a polygonal loop with folds. Because $B$ is compact, its image $\bar{\Phi}(B)$ is a compact domain in $\mathbb{R}^{2}$. Consider an edge whose image contains points belonging to the boundary of $\bar{\Phi}(B)$. Then that edge belongs to an immersed piecewise linear loop with folds $\gamma:[0, n] \rightarrow \mathbb{R}^{2}$ that is the image of one component of $\partial B$.

For some indexing of the edges, there are real numbers $a, b$ such that $[a, b] \subset[0, n]$ and $\left.\gamma\right|_{[a, b]}$ is an embedded loop. Then $\left.\gamma\right|_{[a, b]}$, with its orientation or the reverse, bounds a disk inside the image of $\bar{\Phi}$. Because part of the image of $\gamma_{[a, b]}$ belongs to $\partial \Phi(B)$, it must be $\left.\gamma\right|_{[a, b]}$ with its induced orientation that bounds this disk.

Let $\lfloor a\rfloor$ denote the greatest integer less than or equal to $a$, and $\lceil b\rceil$ denote the least integer greater than or equal to $b$. Then the positive turning of the path $\left.\gamma\right|_{[\lfloor a\rfloor,\lceil b\rceil]}$ is greater than $\pi$. The positive turning along the entire loop $\gamma$ must be at least as large, and an integer multiple of $\pi$. Therefore, it is at least $2 \pi$. 
If $g=0$ then the base is a disk with $k$ holes for some $k \geq 0$. Choose a component of the boundary of the orbit space on which the total turning is at least $2 \pi$. Then use the proof of Lemma 4.9 to construct an embedded right polygonal loop with folds representing the slope list for that boundary component. This polygonal loop bounds a disk $D$ in $\mathbb{R}^{2}$. Remove $k$ disjoint closed disks from the interior of $D$ so as to create $k 1$-holes. Finally, glue in $k$ 1-plugs, the boundaries of which realize the remaining cyclic slope lists encoded in the weighted orbit space.

Meanwhile, the punctured torus example in Example 1.17 generalizes to give immersed examples for any $g$ and any $k \geq 1$, and hence to give examples of toric nearsymplectic manifolds with these orbit spaces.

A complete answer to the question of what $T^{2}$-manifolds admit near-symplectic structures with respect to which the actions are Hamiltonians is not available, but is under investigation by the second author. However, in the next section we prove Theorem 1.14 which provides a complete answer to an intermediate question: Given a $T^{2}$-action, when does there exist a near-symplectic form with respect to which the action is symplectic, and Hamiltonian in a neighborhood of any orbit?

\section{Locally toric near-symplectic manifolds}

As we have seen, an integral affine surface $(B, \mathcal{A}, F)$ defines a toric near-symplectic manifold up to orbit preserving symplectomorphism if and only if its boundary is right polygonal with folds and there exists an integral affine immersion $\Phi:(B \backslash F, \mathcal{A}) \rightarrow$ $\left(\mathbb{R}^{2}, \mathcal{A}_{0}\right)$.

For the purposes of studying pseudo-holomorphic curves in a symplectic 4-manifold via 1-complexes in a surface, the presence of a global torus action is not necessary. All one needs is a singular Lagrangian fibration ${ }^{2}$ in which the behavior of pseudo-holomorphic curves in the neighborhood of each singular fiber is understood. Locally toric fibrations constitute a convenient class of manifolds for this purpose. When the total space is symplectic, the list of manifolds that admit locally toric fibrations is short [13]. However, as we show in this section, there is a vast set of near-symplectic examples.

The following lemma implies that the base of a locally toric fibration is, like a toric fibration, equipped with a natural integral affine structure.

Lemma 5.1 ([18]). Consider the Lagrangian fibration $\pi:\left(\mathbb{R}^{2} \times T^{2}, d p \wedge d q\right) \rightarrow \mathbb{R}^{2}$ in which the map $\pi$ forgets the torus factor. Suppose $U$ is a connected open subset of $\mathbb{R}^{2}$. Then an embedding $\Phi: \mu^{-1}(U) \rightarrow \mathbb{R}^{2} \times T^{2}$ is a fiber-preserving symplectic embedding if and only if it is of the form $(p, q) \mapsto\left(A p+b, A^{-T} q+f(p)\right)$, where $A \in G L(2, \mathbb{Z})$, $b \in \mathbb{R}^{2}, A^{-T}$ is the inverse transpose of $A$, and $f: U \rightarrow T^{2}$ is a smooth map such that $A^{T} \circ D f$ is symmetric.

\footnotetext{
2 Loosely, a singular Lagrangian fibration is a symplectic manifold $(X, \omega)$ together with a projection to a half-dimensional space $B$ such that over a dense open subset of $B$ the projection defines a locally trivial fibration, each of whose fibers is Lagrangian.
} 
Of course, the symplectic manifold $\left(\mathbb{R}^{2} \times T^{2}, d p \wedge d q\right)$ also supports the torus action $t \cdot(p, q)=(p, q+t)$ whose moment map is $\pi$, so the equivariant symplectomorphic embeddings are precisely those fiber-preserving embeddings with $A=\mathrm{Id}$.

Theorem 5.2. Suppose $(B, \mathcal{A}, F)$ is an integral affine surface with right polygonal boundary with edge folds. Then $(B, \mathcal{A}, F)$ is the integral affine base of a locally toric near-symplectic manifold.

Proof. Cover $B$ by a union of open sets $\left\{U_{\alpha}\right\}$, each of which is contractible. Then for each $\alpha$ there exists an integral affine immersion $\Phi_{\alpha}:\left(U_{\alpha} \backslash F, \mathcal{A}\right) \rightarrow\left(\mathbb{R}^{2}, \mathcal{A}_{0}\right)$ and hence a toric near-symplectic manifold $\left(X_{\alpha}, \omega_{\alpha}, \sigma_{\alpha}\right)$ with integral affine base $\left(U_{\alpha}, \mathcal{A}, F\right)$, constructed as in the proof of Theorem 3.5 By Lemma 2.3. on overlaps $U_{\alpha} \cap U_{\beta}$, the two toric near-symplectic manifolds $\left(X_{\alpha}, \omega_{\alpha}, \sigma_{\alpha}\right)$ and $\left(X_{\beta}, \omega_{\beta}, \sigma_{\beta}\right)$ are orbit-preserving symplectomorphic (since $U_{\alpha} \cap U_{\beta}$ must be homeomorphic to a union of contractible spaces). On triple intersections these gluing maps will be compatible, thereby yielding a locally toric near-symplectic manifold.

The only obstruction to the existence of a smooth global torus action inducing a locally toric fibration is monodromy.

Definition 5.3. An integral affine structure $\mathcal{A}$ on a surface $B$ determines a lattice $\Lambda(\mathcal{A})$ in $T B$ (coming via the defining atlas for $\mathcal{A}$ from the standard integral lattice in $\mathbb{R}^{2}$ ). The monodromy of $\mathcal{A}$ is the monodromy representation $\pi_{1}(B) \rightarrow G L(2, \mathbb{Z})$ of $\Lambda(\mathcal{A})$.

Note that this is not the only obstruction if one requires the global action to be Hamiltonian. For example, consider the square $\left\{\left(p_{1}, p_{2}\right)|| p_{i} \mid \leq 1\right\} \subset\left(\mathbb{R}^{2}, \mathcal{A}_{0}\right)$ and identify the top and bottom edges to form an integral affine cylinder. The failure of this integral affine cylinder to isometrically immerse in $\left(\mathbb{R}^{2}, \mathcal{A}_{0}\right)$ implies, by Theorem 3.5 , that it cannot be the integral affine base for a toric fibration.

In the locally toric setting, we do not always have uniqueness of the locally toric manifolds with a given integral affine base.

Theorem 5.4. An integral affine surface whose boundary is right polygonal with edge folds, $(B, \mathcal{A}, F)$, defines a unique locally toric near-symplectic manifold if and only if $B$ is either noncompact or has nonempty boundary (i.e. has the homotopy type of a 1-complex). The uniqueness is up to fiber-preserving homeomorphism that is a symplectomorphism on the symplectic locus.

Note that the base $B$ need not be orientable.

Proof. If $B$ has the homotopy type of a 1-complex then we can find in $B$ a collection $\left\{\gamma_{\alpha}\right\}$ of disjoint properly embedded arcs in $B \backslash F$ such that $B \backslash \bigcup_{\alpha} \gamma_{\alpha}$ is a disjoint union of simply connected surfaces. Choose disjoint open collar neighborhoods $\left\{V_{\alpha}\right\}$ of the $\left\{\gamma_{\alpha}\right\}$ such that for each $\alpha, V_{\alpha} \subset B \backslash F$. Define open sets $\left\{U_{\beta}\right\}$ such that each $U_{\beta}$ is the union of one component of $B \backslash \bigcup_{\alpha} \gamma_{\alpha}$ and all of the $V_{\alpha}$ that have nonempty intersection with that component. Arrange that each $U_{\beta}$ is simply connected by going back and including more arcs in the set $\left\{\gamma_{\alpha}\right\}$ if necessary. 
By Lemma 3.6, each of these integral affine surfaces $\left(U_{\beta} \backslash F, \mathcal{A}\right)$ immerses isometrically in $\left(\mathbb{R}^{2}, \mathcal{A}_{0}\right)$ and hence defines a unique toric near-symplectic manifold, say $\left(X_{\beta}, \omega_{\beta}, \sigma_{\beta}\right)$. Then all of the locally toric near-symplectic manifolds defined by $(B, \mathcal{A}, F)$ can be built out of the $\left(X_{\beta}, \omega_{\beta}, \sigma_{\beta}\right)$ by gluing maps between neighborhoods that project to the $V_{\alpha}$. By Lemma 5.1, each of these maps can be expressed, in local coordinates on the top-dimensional fibers, as $(p, q) \mapsto\left(A p+b, A^{-T} q+\phi(p)\right)$ for some $(A, b) \in \operatorname{Aff}(2, \mathbb{Z})$ and some $\phi(p)$ that is the time-one flow of a Hamiltonian vector field. The element $(A, b) \in \operatorname{Aff}(2, \mathbb{Z})$ is uniquely determined by the integral affine structure on $B$, while the arguments in the proof of Theorem 3.7 show that particular choices of $\phi$ have no effect on the global structure. Thus the global structure defined by $(B, \mathcal{A}, F)$ is unique.

The hypothesis that $B$ have the homotopy type of a 1-complex is necessary because there exist closed integral affine surfaces that each are the integral affine base of more than one locally toric manifold. This is true even if $H^{2}(B, \mathbb{Z})=0$ as evidenced by the existence of multiple locally toric manifolds whose integral affine base is a single integral affine Klein bottle, as shown in [13].

We now take up the problem of realizing $T^{2}$-manifolds as locally toric with respect to some near symplectic structure, i.e. constructing integral affine surfaces with right polygonal boundary that define the underlying fibration.

Lemma 5.5. For any $g \geq 1$ and $k \geq 1$, there exists a noncompact integral affine surface $B$ with empty boundary, genus $g$ and $k$ ends, each of which is a $q$-hole for some $q$ (not necessarily the same $q$ for each end).

Proof. For an appropriately chosen positive integer $p$, let $R$ be a $p$-by-1 rectangle in $\mathbb{R}^{2}$ with quarter- and half-circles, all of radius $r<1 / 2$, removed at the corners of the $p 1$-by-1 rectangles making up $R$. Use $R$ as a fundamental domain to build $B$, gluing appropriate edges to each other via translations. (Without removing the quarter- and half-circles this is a method to produce a flat metric on a closed genus $g$ surface with $k$ singular points; the first author learned this trick from A. Abrams, who claims to have learned it from [21].) Note that, to avoid monodromy, the left edge must be glued to the right, and edges on the top must be glued to edges on the bottom. Figure 6 shows an example for $g=2, k=2$, with one end having $q=1$ and the other having $q=3$.

Lemma 5.6. Given any slope $s \in \mathbb{Q} \cup \infty$, there exists an integral affine annulus with trivial monodromy, with each boundary consisting of a single edge with slope s.

Proof. Glue two sides of a parallelogram via a translation.

Lemma 5.7. Given two right polygonal cyclic slope lists, one of which has at least two slopes, there exists an integral affine annulus with trivial monodromy whose boundary realizes the two slope lists.

Proof. Let the slope lists be $s_{1}, \ldots, s_{k}$ and $t_{1}, \ldots, t_{l}$. If one list has only one slope, then let $\tau$ be a translation in the direction of this slope such that $\left(x_{0}, y_{0}\right):=\tau((0,0))$ is in 


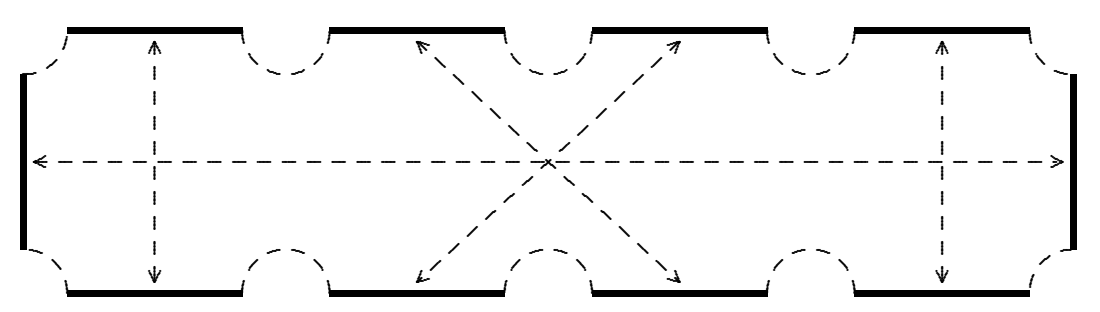

Fig. 6. A fundamental domain for a genus 2 surface with holes; arrows indicate gluing rules.

the closed right half-plane. Otherwise choose such a translation $\tau$ arbitrarily. If $x_{0}, y_{0}>$ 0 then construct, as in the proof of Lemma 4.8. two right polygonal paths with folds representing $s_{1}, \ldots, s_{k}, s_{1}$ and $t_{1}, \ldots, t_{l}, t_{1}$ starting at $(0,0)$ and ending at $\left(x_{0}, y_{0}\right)=$ $\tau((0,0))$. If $x_{0}>0$ and $y_{0} \leq 0$, then construct the path with endpoint $\left(x_{0}, y_{1}\right)$ for some $y_{1}>0$ and lengthen the edge $e_{2}$ so as to lower the endpoint from $y_{1}$ to $y_{0}$. If $x_{0}=0$, then interchange the roles of $x$ and $y$ (thereby affecting the slopes also), make the construction, and switch back.

Construct two $\tau$-invariant periodic paths by concatenating translated copies of these paths. Translate one of the periodic paths so that they become disjoint, and rotate one by $180^{\circ}$ so that they are the oriented boundary of the strip $S$ in between. Then $S /\langle\tau\rangle$ is a base with folds homeomorphic to an annulus with boundary realizing the two slope lists.

Lemma 5.8. Given three slopes $s_{1}, s_{2}, s_{3} \in \mathbb{Q} \cup\{\infty\}$, there exists an integral affine twice-punctured disk with trivial monodromy with each boundary component consisting of a single edge of slope $s_{i}$.

Proof. If two of the slopes are equal, use Lemma 5.6 with one 1-hole, and fill with a plug from Lemma 4.17 Otherwise, without loss of generality we can assume that $s_{1}<$ $0=s_{2}<s_{3}<\infty$ (recall that the order is unimportant). Figure 7 then illustrates the construction by means of a fundamental domain embedded in $\mathbb{R}^{2}$.

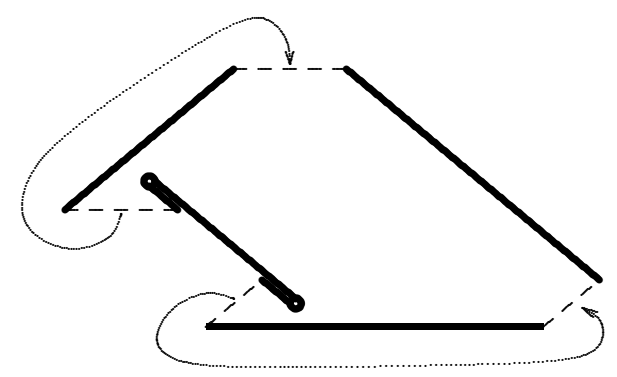

Fig. 7. Building a twice-punctured disk with one edge per boundary. 
Proof of Theorem 1.14 Theorems 5.2 and 5.4 reduce our proof to proving that, given a genus $g$ and $k$ slope lists, there exists an immersion of a genus $g$ surface with $k$ boundary components - or a fundamental domain of such a surface-into $\mathbb{R}^{2}$ so that its boundary is a union of right polygonal loops with folds realizing the prescribed slope lists if and only if the hypotheses of the theorem are satisfied.

Notice that since the union of principal orbits is an oriented $T^{2}$-bundle, the integral affine structure must have trivial monodromy. Accordingly, the gluing maps used to construct the surface from an immersion of a fundamental domain must be translations.

We now go through the various cases of specific genera and numbers of boundary components.

If the surface has no boundary, then the presence of an integral affine structure implies that the tangent bundle admits a flat connection, and therefore has zero Euler characteristic. Since the monodromy is trivial, the surface must be orientable, and therefore a torus. Any torus bundle over a torus with trivial monodromy (but not necessarily trivial Chern class) supports a near-symplectic structure with respect to which the action is locally toric. Since the boundary is empty, the near-symplectic structure is in fact symplectic. Such manifolds were classified by Mishachev [15]. The bases of such locally toric symplectic manifolds can be constructed by choosing a parallelogram in the plane that is integral affine equivalent to a rectangle, and pairwise identifying the opposite edges via translations.

Now suppose the surface has nonempty boundary. We first suppose that $g \geq 1$. Then Lemma 5.5 tells us how to construct a noncompact integral affine surface (without boundary) with $k$ ends, and Lemma 4.17 asserts that we can glue into each of these ends the collar neighborhood of a boundary component that realizes any given slope list.

If the surface has genus zero we have three cases to consider: $k \geq 3, k=2$, and $k=1$.

Suppose $k \geq 3$. If all of the slope lists consist of exactly one slope, we can use Lemma 5.8 and any three of the slopes to construct a punctured annulus realizing these three slopes, and then remove neighborhoods of slits and use Lemma 4.17 to fill in the remaining boundary components. Otherwise, Lemma 5.7 tells us how to construct an annulus realizing two of the slope lists, one of which has at least two slopes; then again, we can remove disks to create 1-holes and then glue in 1-plugs realizing the remaining slope lists.

If $k=2$ and one slope list has at least two slopes (which happens if and only if $T_{0} \geq \pi$ ) then we can use Lemma 5.7 together with 1-plugs from Lemma 4.17 to construct the required immersed surface. Otherwise, if both slope lists consist of just one slope, then we merely need to invoke Lemma 5.6. In this latter case $T_{0}=0$. Notice that the two slopes must be equal for otherwise we would be trying to glue the two parallel sides of a trapezoid via a translation-which we can do only if they have the same length, i.e. if the trapezoid is actually a rectangle.

For the last case, suppose $k=1$. In this case the weighted orbit space is simply connected. Therefore given any integral affine structure $\mathcal{A}$ on the orbit space, there is an integral affine immersion $(B, \mathcal{A}) \rightarrow\left(\mathbb{R}^{2}, \mathcal{A}_{0}\right)$. Consequently, if the action is locally 
Hamiltonian it must be Hamiltonian. Therefore, the necessary and sufficient conditions are contained in Theorem 1.12 .

Lastly, to compute the number of components of the vanishing locus, note that $\left|Z_{\omega}\right|=$ $|F|$, the number of folds. The total turning on all boundaries of the base is $T-|F| \pi$, where $T$ is the sum of the positive turnings over all boundary components. The Gauss-Bonnet Theorem implies that $2 \pi \chi=T-\pi|F|$, and hence $\left|Z_{\omega}\right|=T / \pi-2 \chi$.

We now forget about torus actions. In order to highlight the plenitude of closed locally toric near-symplectic manifolds we prove Propositions 1.15 and 1.16 . Recall that the first proposition asserts that any monodromy representation of the fundamental group of any surface can arise as the monodromy of a locally toric fibration of a closed near-symplectic manifold, while the second asserts that there is an infinite family of mutually nondiffeomorphic closed near-symplectic manifolds that support locally toric fibrations having nontrivial monodromy, none of which could support a locally toric fibration with trivial monodromy.

Proof of Proposition 1.15 Suppose the free group has $n$ generators $x_{1}, \ldots, x_{n}$, and the homomorphism maps $x_{i}$ to the matrix $\left(\begin{array}{cc}a_{i} & c_{i} \\ b_{i} & d_{i}\end{array}\right) \in G L(2, \mathbb{Z})$. Draw a (probably nonconvex) polygon with at least $3 n$ edges in $\mathbb{R}^{2}$ having rational slope, such that at every vertex the two incident edges have primitive integral tangent vectors $v, w \operatorname{satisfying} \operatorname{det}(v, w)=$ \pm 1 . Draw the polygon so that it has $2 n$ distinguished edges $e_{1}, \ldots, e_{n}$ and $f_{1}, \ldots, f_{n}$, satisfying the following properties:

(1) The primitive integral tangent vector to each $e_{i}$ is $\left(\begin{array}{l}1 \\ 0\end{array}\right)$, while the primitive integral tangent vector to the edge immediately preceding $e_{i}$ (with the boundary orientation) is $\left(\begin{array}{c}0 \\ -1\end{array}\right)$ and the primitive integral tangent vector to the edge immediately following $e_{i}$ (with the boundary orientation) is $\left(\begin{array}{l}0 \\ 1\end{array}\right)$.

(2) The primitive integral tangent vector to each $f_{i}$ is $\left(\begin{array}{c}a_{i} \\ b_{i}\end{array}\right)$, while the primitive integral tangent vector to the edge immediately preceding $f_{i}$ (with the boundary orientation) is $\left(\begin{array}{c}c_{i} \\ d_{i}\end{array}\right)$ and the primitive integral tangent vector to the edge immediately following $f_{i}$ (with the boundary orientation) is $\left(\begin{array}{c}-c_{i} \\ -d_{i}\end{array}\right)$.

(3) The lengths of the $e_{i}$ and $f_{i}$ are chosen so that, for each $i$, the Euclidean lengths of $\left(\begin{array}{ll}a_{i} & c_{i} \\ b_{i} & d_{i}\end{array}\right) e_{i}$ and $f_{i}$ are equal.

(4) As one traverses the boundary of the polygon counterclockwise, the edges $e_{i}$ and $f_{i}$ are ordered so that identification of each $e_{i}$ with each $f_{i}$ produces the surface $B$.

Now construct a base with folds from this polygon by replacing each concave corner with a convex corner and a nearby fold (extend one incident edge a little past the corner and then immediately double back, creating a fold point, then turn onto the other incident edge) and by gluing a rectangular neighborhood of $e_{i}$ to a rectangular neighborhood of $f_{i}$ via $\left(\begin{array}{cc}a_{i} & c_{i} \\ b_{i} & d_{i}\end{array}\right)$ followed by a suitable translation.

Proof of Proposition 1.16. Figure 8 gives the construction of the infinite family, where the ellipses in the middle are to be interpreted as representing $n$ "slits." Call these manifolds $X_{n}, n \geq 0$.

These manifolds are mutually nondiffeomorphic because adding a slit has the effect of adding a generator and no relations to the fundamental group of the 4-manifold. 


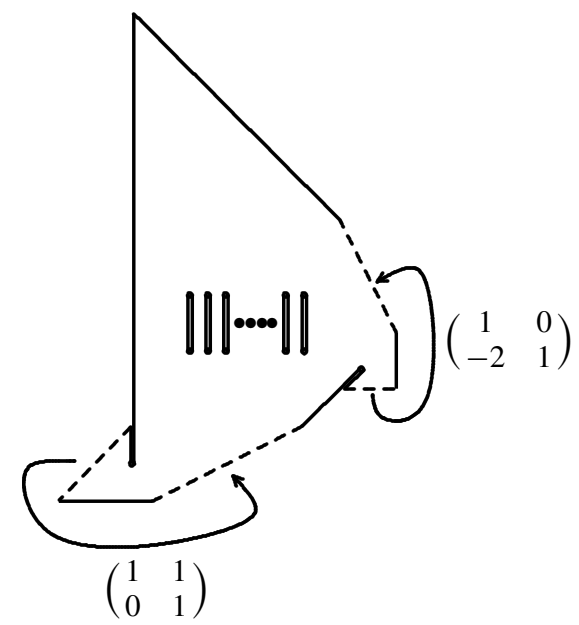

Fig. 8. An infinite family of examples with nontrivial monodromy. (All lines in this figure have slope $0, \pm 1 / 2, \pm 1, \pm 2$ or $\infty$.)

To see why no $X_{n}$ can be diffeomorphic to a locally toric near-symplectic manifold whose fibration has trivial monodromy we note that, by Lemma $4.13, \chi\left(X_{n}\right)=1$ for all $n$. However, a near-symplectic manifold equipped with a locally toric fibration having trivial monodromy can never have Euler characteristic equal to 1 . Indeed, this would imply that the integral affine base would have a boundary component with just one vertex. With trivial monodromy, this means that the isotropy subgroups for orbits whose images are on either side of the vertex are the same, violating the requirement that the boundary of the integral affine base have right polygonal boundary.

\section{Appendix. Hamiltonian actions}

In this section we prove Proposition 1.2 which gives a simplified definition of a Hamiltonian action when the group is a torus (what we called a "topologist's definition").

In general, given a group action $\sigma: G \times X \rightarrow X$ on a symplectic manifold $(X, \omega)$, there are two types of natural vector fields. For each $\xi$ in the Lie algebra $\mathcal{G}$ there is the infinitesimal action $V_{\xi}$, while for each smooth function $f: X \rightarrow \mathbb{R}$ there is the Hamiltonian vector field $V_{f}$ defined by $\omega\left(V_{f}, \cdot\right)=-d f$.

Definition A.1. A group action $\sigma: G \times X \rightarrow X$ on a symplectic manifold $(X, \omega)$ is Hamiltonian if there is a Lie algebra homomorphism from $\mathcal{G}$ to $C^{\infty}(X)$ (equipped with the Poisson bracket) that sends $\xi$ to the function $f_{\xi}$ so that $V_{f_{\xi}}=V_{\xi}$.

Proof of Proposition 1.2 When the group is a torus $T^{n}, \mathcal{G}=\mathcal{G}^{*}=\mathbb{R}^{n}$. In that case, Definition A.1 implies the existence of a moment map $\mu: M \rightarrow \mathcal{G}^{*}$ defined implicitly by 
$\langle\mu(x), \xi\rangle=f_{\xi}(x)$. Since $d f_{\xi}(W)=\xi \cdot D \mu(W)$ for any vector $W$, one direction of the proposition is clear.

For the converse, the functions $f_{\xi}$ are just $\xi \cdot \mu$. We only need to check that $\xi \mapsto f_{\xi}$ is a Lie algebra homomorphism. Since the action is abelian, for any $\xi, \eta \in \mathbb{R}^{2}=\mathcal{T}$ we have $[\xi, \eta]=0$. Therefore, $\xi \mapsto f_{\xi}$ will be a Lie algebra homomorphism provided $\left\{f_{\xi}, f_{\eta}\right\}=\omega\left(V_{\xi}, V_{\eta}\right)=0$.

First of all, for any $\xi, \eta, \omega\left(V_{\xi}, V_{\eta}\right)$ is constant on the orbit of $V_{\eta}$. This follows from two facts: the time- $t$ flow $\phi_{t}$ of $V_{\eta}$ is a symplectomorphism for each $t \in \mathbb{R}$ and the action is abelian so $\left(\phi_{t}\right)_{*} V_{v}=V_{v} \circ \phi_{t}$ for each $v \in \mathcal{T}$. Calculating $\omega\left(V_{\xi}, V_{\eta}\right)=-d f_{\xi}\left(V_{\eta}\right)=$ $-V_{\eta} f_{\xi}$ we see that $V_{\eta} f_{\xi}$ is constant on the orbit of $V_{\xi}$. For almost all $\eta$, the orbit is a circle, and hence the derivative $V_{\eta} f_{\xi}$, being equal to a constant, must be equal to zero, implying that $f_{\xi}$ is constant on the orbit of $V_{\eta}$. Continuity of the moment map then implies that this is true for all $\eta$, thereby establishing that $\omega\left(V_{\xi}, V_{\eta}\right)=0$ for all $\xi, \eta$.

Remark A.2. The compactness of the torus is essential here. Note that there is a symplectic action of $\mathbb{R}^{2}$ on $\left(\mathbb{R}^{2}, \omega_{0}\right)$, by translation, that satisfies the "topologist's definition" (Proposition 1.2) but is certainly not Hamiltonian.

\section{References}

[1] Arnol'd, V. I.: Mathematical Methods of Classical Mechanics. 2nd ed., Grad. Texts in Math. 60, Springer, New York (1989) Zbl 0386.70001 MR 1345386

[2] Audin, M.: Torus Actions on Symplectic Manifolds. Rev. ed., Progr. Math. 93, Birkhäuser, Basel (2004) Zbl 1062.57040 MR 2091310

[3] Auroux, D., Donaldson, S. K., Katzarkov, L.: Singular Lefschetz pencils. Geom. Topology 9 , 1043-1114 (2005) Zbl 1077.53069 MR 2140998

[4] Delzant, T.: Hamiltoniens périodiques et images convexes de l'application moment. Bull. Soc. Math. France 116, 315-339 (1988) Zbl 0676.58029 MR 0984900

[5] Fintushel, R., Stern, R. J.: Knots, links, and 4-manifolds. Invent. Math. 134, 363-400 (1998) Zbl 0914.57015 MR 1650308

[6] Gay, D. T., Kirby, R.: Constructing symplectic forms on 4-manifolds which vanish on circles. Geom. Topology 8, 743-777 (2004) Zbl 1054.57027 MR 2057780

[7] Gompf, R. E.: A new construction of symplectic manifolds. Ann. of Math. (2) 142, 527-595 (1995) Zbl 0849.53027 MR 1356781

[8] Honda, K.: An openness theorem for harmonic 2-forms on 4-manifolds. Illinois J. Math. 44, 479-495 (2000) Zbl 0970.58001 MR 1772422

[9] Honda, K.: Local properties of self-dual harmonic 2-forms on a 4-manifold. J. Reine Angew. Math. 577, 105-116 (2004) Zbl 1065.53066 MR 2108214

[10] Kaufman, S.: Delzant-type classification of near-symplectic toric 4-manifolds. arXiv:math. SG/0505369

[11] Lerman, E.: Symplectic cuts. Math. Res. Lett. 2, 247-258 (1995) Zbl 0835.53034 MR 1338784

[12] Lerman, E., Tolman, S.: Hamiltonian torus actions on symplectic orbifolds and toric varieties. Trans. Amer. Math. Soc. 349, 4201-4230 (1997) Zbl 0897.58016 MR 1401525

[13] Leung, N. C., Symington, M.: Almost toric symplectic four-manifolds. arXiv:math. $\mathrm{SG} / 0312165$ 
[14] Mikhalkin, G.: Enumerative tropical algebraic geometry in $\mathbb{R}^{2}$. J. Amer. Math. Soc. 18, 313377 (2005) Zbl 1092.14068 MR 2137980

[15] Mishachev, K. N.: The classification of Lagrangian bundles over surfaces. Differential Geom. Appl. 6, 301-320 (1996) Zbl 0921.58018 MR 1422337

[16] Orlik, P., Raymond, Frank: Actions of the torus on 4-manifolds. I. Trans. Amer. Math. Soc. 152, 531-559 (1970) Zbl 0216.20202 MR 0268911

[17] Parker, B.: Holomorphic curves in lagrangian torus fibrations. Ph.D. thesis, Stanford Univ. (2005)

[18] Symington, M.: Four dimensions from two in symplectic topology. In: Topology and Geometry of Manifolds (Athens, GA, 2001), Proc. Sympos. Pure Math. 71, Amer. Math. Soc., Providence, RI, 153-208 (2003) Zbl 1049.57016 MR 2024634

[19] Taubes, C. H.: Seiberg-Witten invariants and pseudo-holomorphic subvarieties for self-dual, harmonic 2-forms. Geom. Topology 3, 167-210 (1999) Zbl 1027.53111 MR 1697181

[20] Taubes, C. H.: A compendium of pseudoholomorphic beasts in $\mathbb{R} \times\left(S^{1} \times S^{2}\right)$. Geom. Topology 6, 657-814 (2002) Zbl 1021.32008 MR 1943381

[21] Thurston, W. P.: On the geometry and dynamics of diffeomorphisms of surfaces. Bull. Amer. Math. Soc. (N.S.) 19, 417-431 (1988) Zbl 0674.57008 MR 0956596

[22] Thurston, W. P.: Three-Dimensional Geometry and Topology. Vol. 1. Princeton Math. Ser. 35, Princeton Univ. Press, Princeton, NJ (1997). Zbl 0873.57001 MR 1435975 